\title{
Revisión sistemática (2010-2017) de los instrumentos de evaluación y programas de rehabilitación de la cognición social utilizados con personas con esquizofrenia.*
}

\section{Systematic Review (2010-2017) of evaluation instruments and rehabilitation programs of social cognition, used with people with schizophrenia.}

\author{
Margarita Loubat \\ Escuela de Psicología Universidad de Santiago de Chile, Chile \\ Albert Astudillo-Zúñiga \\ Hospital Padre Hurtado, Chile \\ (Recibido: 02 de enero de 2018) (Aceptado: 27 de noviembre de 2018)
}

\begin{abstract}
Resumen
Búsqueda de instrumentos de evaluación y programas de entrenamiento de la cognición social (CS) en beneficio de la rehabilitación del paciente con esquizofrenia. Se utiliza la metodología Prisma realizando una revisión en bases de datos Scopus, ISI y ApaPsycNet, años 2010-2017, considerando como criterio de inclusión investigaciones donde se utilizan instrumentos con fines evaluativos y programas de rehabilitación de los diversos subdominios de la CS, para personas con esquizofrenia. Se ubican 290 artículos y se seleccionan 195 , los cuales se revisan en su totalidad. Se establece una síntesis de 37 instrumentos y se describen 10 programas de rehabilitación de la CS. Se concluye en la necesidad de explicitar mayormente en la literatura científica las propiedades, fiabilidad y validez de los test utilizados a fin de medir lo que se desea medir. También, describir los programas utilizados en beneficio de la rehabilitación de la CS del paciente con esquizofrenia. Palabras clave: cognición social, instrumentos de evaluación, programas de rehabilitación, esquizofrenia.
\end{abstract}

\begin{abstract}
Research is done regarding evaluation instruments and training programs of social cognition in benefit of the rehabilitation processes of patients with schizophrenia.The PRiSM methodology is used, reviewing the Scopus, ISI and ApaPsycNet journals databases, between 2010 and 2017, considering as inclusion criteria pieces of research where instruments with evaluative purposes and rehabilitation programs of the different sub-domains of social cognition for people with schizophrenia are used. 290 articles are found, and 195 which are thoroughly examined, are selected. A summary of 37 instruments is established, and 10 rehabilitation programs of social cognition are described.It is concluded that it is necessary to explicitly state in the literature the properties, reliability and validity of the tests used, so as to measure what is intended to be measured. Also, it is important to describe the programs used in benefit of the social cognition rehabilitation of the patient with schizophrenia.

Key words: social cognition, evaluation instruments, rehabilitation programs, schizophrenia
\end{abstract}

Correspondencia: Margarita Loubat, margarita.loubat@usach.cl

*Este estudio fue financiado por el Proyecto DICYT N031793LO, de la Dirección de Investigación Científica y Tecnológica de la Universidad de Santiago de Chile. 


\section{Introducción}

Uno de los objetivos, si no el principal, de la rehabilitación en personas con trastornos mentales de curso crónico es restaurar y/o desarrollar habilidades de carácter social, con el propósito que logren una mejor vinculación con sus familias, redes de apoyo y puedan acceder a reinsertarse socialmente.

Para esos efectos la cognición social (CS) juega un papel fundamental, pues está vinculada a la manera cómo las personas se relacionan con su entorno. La CS es un proceso cognitivo que permite elaborar conductas sociales adaptativas y que consta -según algunos autores- de procesos básicos denominados dominios. Forman parte de ellos el Procesamiento emocional, que incluye la percepción y reconocimiento de las emociones a partir de expresiones faciales e inflexión de la voz; el Conocimiento social, que se refiere al entendimiento que una persona tiene sobre roles y reglas sociales de su cultura; la Percepción social, que implica la capacidad de juzgar y decodificar roles y contextos sociales; el Estilo atribucional, que se refiere a la forma particular que tiene una persona al explicar las causas de los hechos de su vida; y, la Teoría de la mente (ToM), la cual hace referencia a la capacidad para hacer inferencias sobre los estados mentales de otros, como por ejemplo: intenciones, disposiciones y creencias ajenas (Brüne, 2005; Zabala, Richard's, Breccia \& López, 2018).

Estas operaciones mentales son procesos que se activan en contextos de interacción social y que permiten percibir, evaluar y responder adecuadamente a situaciones considerando y valorando las propias impresiones como infiriendo las de los otros. Son procesos naturales en la mayoría de las personas, pero en sujetos con esquizofrenia (EQZ) se encuentran profundamente dañados. En efecto, cuando esos procesos no se han desarrollado convenientemente pueden derivar en percepciones sociales incorrectas, respuestas inadecuadas e incluso, provocar un aislamiento social en quienes padecen EQZ, con implicancias en la calidad de vida y en la tasa de recaídas, considerando que la habilidad para procesar rápidamente estímulos sociales, es esencial en las interacciones sociales (White, Laithwaite \& Gilbert., 2013; Torgalsbøen, Mohn \& RishovdRund, 2014). A ello se agrega, que cuando se estudia el impacto de las capacidades cognitivas sociales y no sociales para el funcionamiento diario de estos pacientes, las capacidades cognitivas no sociales (atención, memoria, función ejecutiva), tienden a perder fuerza explicativa, siendo el deterioro en la cognición social un elemento central en la explicación de esas deficiencias (Billeke \& Aboitiz, 2013).

Parece entonces necesario conocer en detalle las dificultades en CS de quienes padecen psicosis o esquizofrenia, utilizando para ello instrumentos fiables, lo cual permitiría una mejor caracterización de esos problemas y dirigir intervenciones psicoterapéuticas más focalizadas (Derntl, Seidel, Schneider \& Habel, 2012). Ello, sin perder de vista que las alteraciones cognitivas están presentes incluso antes del primer diagnóstico del trastorno, como parte del pródromo, y que tienden a mejorar o mantenerse invariables al estabilizarse la situación clínica o aumentar con las recaídas y el paso de los años (Elsawy, El-Hay \& Badawy, 2010).

Es en base a la evidencia presentada, que el objetivo de este estudio es efectuar una búsqueda de instrumentos de evaluación y de programas de rehabilitación de la CS específicos para sujetos que padecen EQZ, dada la relevancia teórico-práctica que ello reviste para la rehabilitación de la funcionalidad diaria de estas personas.

\section{Metodología}

Se realiza un estudio descriptivo-analítico en base a la metodología PRISMA (Urrútia \& Bonfill, 2010; Santelices et al. 2016), de artículos que incluyen instrumentos y programas de rehabilitación de la CS, en bases de datos de revistas Scopus, ISI y ApaPsycNet, entre los años 2010 y 2017. Como estrategia de búsqueda se asocian las palabras cognición social y esquizofrenia, en español y en inglés, bajo la consideración del título y del resumen del artículo. Se seleccionan artículos que incluyen instrumentos de evaluación y/o programas de rehabilitación de la cognición social en personas con esquizofrenia. Se leen en su totalidad. Los instrumentos se agrupan según la dimensión de la CS que evalúan, se releva el formato que presentan y sus características psicométricas. Cuando en la búsqueda primaria no se explicitan esas características, se realiza una búsqueda en fuentes secundarias. Por último, se filtran y seleccionan aquellos test que muestran explícitamente aspectos psicométricos y que la utilización del instrumento esté condicionada a evaluar lo que se desea medir con fines diagnósticos y/o como evidencia del impacto de un tratamiento determinado. Respecto de los programas, éstos se integran bajo el criterio que se explicite el programa realizado, el subdominio de la CS, el formato y el éxito terapéutico. 


\section{Resultados}

En la Figura 1 se muestra el proceso de revisión efectuado: de un total de 290 artículos encontrados, se seleccionan 195 y se analizan en su totalidad. Finalmente, bajo los criterios de inclusión anteriormente mencionados, se seleccionan 8/26 instrumentos para la dimensión de Procesamiento Emocional; 6/19 de Percepción Social; 6/9 de Conocimiento Social; 5/7 de Estilo de Atribución; 8/23 de Teoría de la Mente; y 4/10 Instrumentos que abarcan varios subdominios de la Cognición Social. A su vez se contabilizan 10 programas de rehabilitación de la CS, según criterios de inclusión.

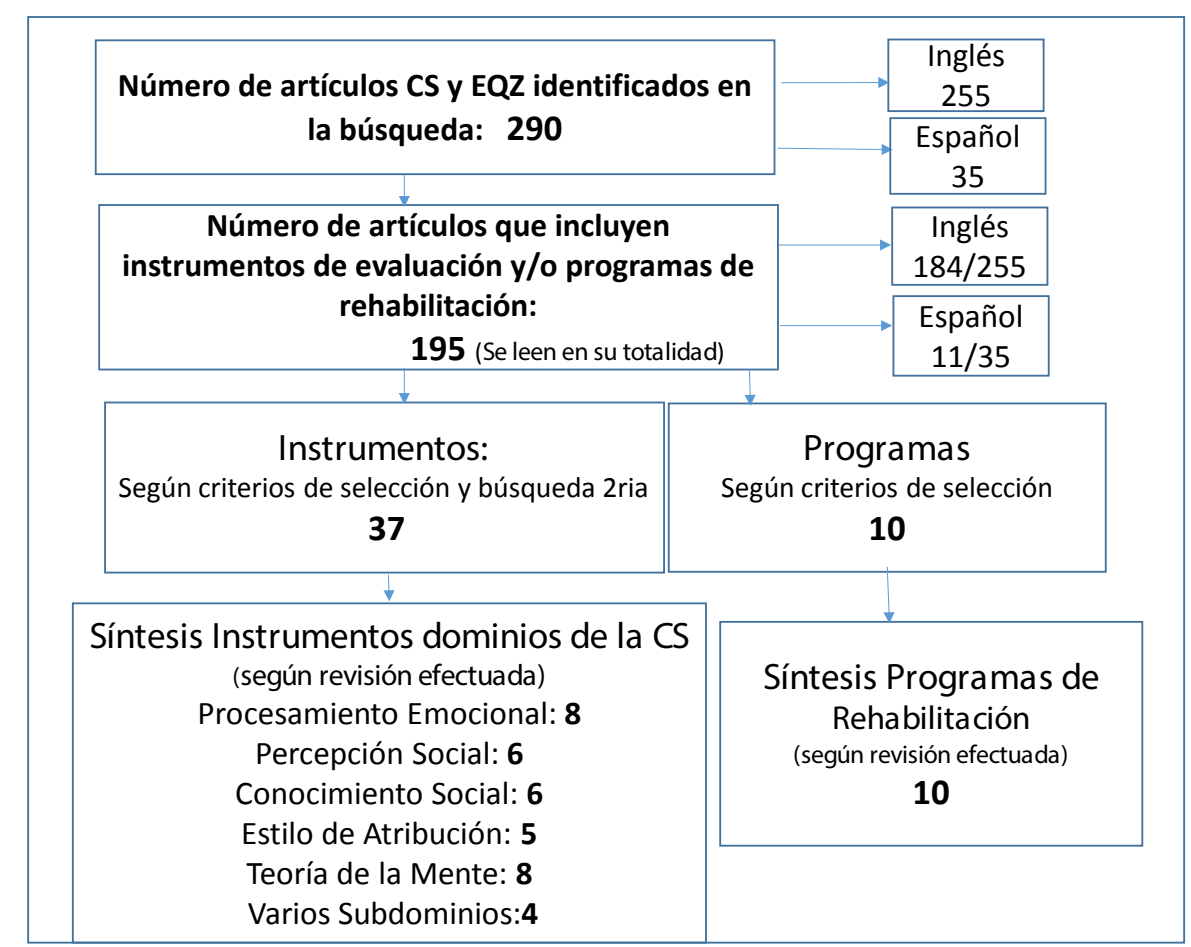

\section{A.- Instrumentos de evaluación de la Cognición Social.}

a. Sub dimensión: Procesamiento Emocional:

Vinculada con percibir y utilizar las emociones (identificación, comprensión y manejo emocional). En la fase crónica se verían más afectado el reconocimiento de rostros y tono de voz; y en población de alto riesgo (pródromo) ya se apreciarían dificultades con la identificación de las emociones (García, Tirapu \& López-Goñi, 2012). 


\section{1.- Instrumentos de evaluación: subdominio Procesamiento Emocional}

\begin{tabular}{lll}
\hline TEST Y AUTOR & FORMATO & ASPECTOS PSICOMÉTRICOS \\
\hline $\begin{array}{l}\text { 1.- Tool for Recognition of Emotions in Neuropsychiatric } \\
\begin{array}{l}\text { Disorders (TRENDS) } \\
\text { (Behere et al, 2008) }\end{array}\end{array}$ & $\begin{array}{l}\text { Imágenes y } \\
\text { videos }\end{array}$ & $\begin{array}{l}\text { Alfa de Cronbach, 0.669 Para imágenes } \\
\text { estáticas, 0.447 para imágenes dinámicas. }\end{array}$ \\
$\begin{array}{l}\text { 2.- Pictures of Facial Affect (POFA) } \\
\text { (Ekman \& Friesen, 1976) }\end{array}$ & Imágenes & Confiabilidad test re-test Spearman: 70 \\
$\begin{array}{l}\text { 3.- Facial Emotion Discrimination Task (FEDT) } \\
\text { (Kerr \& Neale, 1993) }\end{array}$ & $\begin{array}{l}\text { Imágenes, } \\
\text { DVD }\end{array}$ & $\begin{array}{l}\text { Alpha de Cronbach, Paciente agudo=66, } \\
\text { Paciente en tratamiento }=67, \text { Controles } \\
=64\end{array}$
\end{tabular}

4.- The Facial Emotion Identification Task(FEIT) (Kerr \& Neale, 1993)

5.- Emotional Intelligence Test (MSCEIT) (Mayer, Salovey \& Caruso, 2002)

6.- The facial affect recognition test (Poole, Tobias, Vinogradov \& 2000)

7.- Bell-Lysaker Emotion Recognition Task (BLERT) (Bell, Bryson \& Lysaker, 1997)

8.- Ekman 60 Faces Test, Facial Expressions of emotion task (FEEST)(Ekman \& Friesen, 1976)
Imágenes, Alpha de Cronbach Paciente agudo=37, DVD Paciente en tratamiento $=52$, Controles $=$ 41

Escala de Alpha de Cronbach: 0.93 Confiabilidad evaluación test re test: 0.55 a 0.88

Imágenes

Alpha de cronbach $=0.72$

Video

Precisión de un $92 \%$ en muestras normales. Y 77\% en pacientes EQZ., Confiabilidad test retest $\mathrm{R}=0.76$ y estabilidad medida con kappa $=0.93$

Imágenes y Confiabilidad $=0,62$, Validez $=0,81$

Instrumentos citados en: TRENDS: Mehta et al, 2014; POFA: Fernández-Gonzalo et al, 2015; de Achával et al, 2012; Miyata et al, 2010; Yamada et al, 2007. FEDT: Brown et al, 2014; Roberts et al, 2014; Tas et al, 2012; Lindenmayer et al, 2013; Combs et al, 2007; Horton \& Silverstein, 2008; Fuentes et al, 2011. FEIT, Brown et al, 2014; Roberts et al, 2014; Savla et al, 2012; Brekke et al, 2007; Lindenmayer et al, 2013; Rocha \& Queirós, 2013; Combs et al, 2007; Wang et al, 2013; Sergi et al, 2009; Fuentes et al, 2011; Okruszek et al, 2017; GilSanz et al, 2017; Mervis et al, 2017; Palumbo et al, 2017, MSCEIT: Linke et al, 2015; Lin et al, 2013; Minor et al, 2015; Savla et al, 2012; Tso et al 2010; Frajo-Apor, 2016; Lindenmayer et al, 2013; Fanning et al, 2012; Hasson-Ohayon et al, 2015; Rocha \&Queirós, 2013 ; Minor\&Lysaker, 2014; Nitzburg et al, 2015; Horan et al, 2012; Rassovsky et al, 2015. The facial affect recognition: Gard et al, 2009; BLERT: Buck et al, 2014; Minor et al, 2015; Lysaker et al, 2013; Buck et al, 2015; Renard et al, 2012; Fanning et al, 2012; Lysaker 2012; Minor \&Lysaker, 2014; Kalin et al, 2015; Thaler et al, 2014; Lysaker et al, 2013; Browne et al, 2016; FEEST: Guastella et al, 2015; Balogh et al, 2014; Sparks et al, 2010; Whitton\& Henry, 2013; Vázquez-Campo et al, 2016 


\section{2.-Descripción de los instrumentos de la Tabla $N^{\circ} 1$}

1.- Tool for Recognition of Emotions in Neuropsychiatric Disorders, TRENDS. (Behere et al., 2008). Instrumento de origen Indio que evalúa expresión y percepción emocional. Posee dos partes: la primera presenta 52 imágenes estáticas y la segunda 28 imágenes dinámicas, presenta 6 emociones básicas: felicidad, tristeza, miedo, enojo, sorpresa y asco, en intensidad baja y alta. Todas interpretadas por actores.

2.- Pictures of Facial Affect, POFA. (Ekman \& Friesen, 1976). Instrumento destinado al reconocimiento de 6 emociones básicas: felicidad, tristeza, miedo, enojo, sorpresa y asco, en 60 imágenes en blanco y negro, de hombres y mujeres, se puntúa 0 (respuesta incorrecta) o 1 (respuesta correcta) en cada imagen.

3.- Facial Emotion Discrimination Task, FEDT. (Kerr \& Neale, 1993). Test de discriminación facial de emociones. Consta de 30 imágenes en blanco y negro; cada imagen presenta 1 o 2 de las seis emociones básicas, proyectadas de manera simultánea por $15 \mathrm{~s}$ con subsecuentes $15 \mathrm{~s}$ de pantalla en blanco. La tarea consiste en juzgar adecuadamente cuál de los dos rostros presentados corresponde a la emoción indicada.

4.- The Facial Emotion Identification Task, FEIT. (Kerr \& Neale, 1993). Se presentan 19 imágenes que representan 1 de 6 emociones básicas, se muestran 1 por cada $15 \mathrm{~s}$, con 10 s de pantalla en blanco entre cada una. Luego de cada estimulo, el participante debe elegir cuál de las 6 emociones es la representada. El puntaje es la suma total de las respuestas correctas.

5.- Emotional Intelligence Test, MSCEIT. (Mayer et al., 2002). Forma parte de la batería MATRICS y consiste en una evaluación auto-administrada que consta de 8 tareas divididas en cuatro áreas: percepción de emoción, facilitación de emociones, entendimiento de emociones y manejo de emociones. Se responde a 29 ítems en una escala Likert de 5 puntos, donde un puntaje inferior a 40 refleja déficit.

6.- The facial affect recognition test, (Poole et al., 2000). Este instrumento usa fotografías de actores, dos hombres y dos mujeres, dividiéndose en cuatro categorías de emociones: enojo, felicidad, miedo y neutral. Consta de 64 ítems con 16 representaciones por cada categoría, donde el evaluado debe reconocer la emoción. 7.- Bell-Lysaker Emotion Recognition Task, BLERT, (Bell et al., 1997). Instrumento que contiene 21 segmentos de video, el participante debe identificar las emociones que aparecen. Ha sido ampliamente usado en evaluación de la cognición social en esquizofrenia y ha demostrado estabilidad categorial en el tiempo y validez discriminativa en esta población de personas.

8.-Ekman 60 Faces Test, Facial Expressions of emotion task, FEEST. (Ekman \& Friesen, 1976). Evalúa el reconocimiento emocional facial de la alegría, tristeza, enojo, miedo, sorpresa y desagrado, en una serie de 60 imágenes en blanco y negro, 10 por cada emoción, presentadas en computador. Las imágenes se muestran de manera aleatoria y el participante debe indicar la etiqueta emocional que mejor representa la imagen. Este instrumento ha mostrado confiabilidad y es válido para el reconocimiento emocional.

\section{b. Subdimensión: Percepción social}

Capacidad para decodificar e interpretar las claves sociales en los gestos comunicativos de los demás y su comportamiento en un contexto social. A diferencia de la percepción emocional, la percepción social se centra en el contexto, por lo que la información no se limita a las claves emocionales, si no en el manejo de claves multimodales para poder hacer inferencias acerca de situaciones sociales, por ejemplo, ambiguas. 


\section{1.- Instrumentos de evaluación: subdominio Percepción Social.}

\begin{tabular}{lll}
\hline TEST Y AUTOR & FORMATO & ASPECTOS PSICOMÉTRICOS \\
\hline 1.-The Interpersonal Re- & Escala de & Modelo de Davis, estructura interna de la prueba: $\chi^{2}=781.74(344)$, \\
activity Index (IRI). & evaluación & $\mathrm{p}<.001$, RMSEA $=0.054$, CFI $=0.813$, y SRMR $=0.070$. RMSEA \\
(Davis, 1983) & & y SRMR. Modelo de Cliffords on second-order model shown in: $\chi^{2}=$ \\
& $810.34(346), \mathrm{p}<.001, \mathrm{RMSEA}=0.056, \mathrm{CFI}=0.802, \mathrm{y}$ SRR $=0.075$.
\end{tabular}

2.-Observable Social Cognition, A Rating Scale (OSCARS)

(Healey et al., 2015)

3. "Social Responsiveness Scale SRS" (Constantino et al., 2003)

4.- Clinical Evaluation of Language Fundamentals (CELF-5) (Wiig et al., 2013)

5.- Balanced Emotional Empathy Scale (BEES) (Mehrabian,1996)

6.- Empathy Quotient (EQ) (Baron-Cohen, 2004)

\section{Escala de} evaluación

Adaptación al español (Chile)

Consistencia interna Alpha de Cronbach $=80$ en pacientes, 78 en controles. Test re-test confiabilidad en rangos de 50 a 70 (media 62, Desviación estandar 07). Confiabilidad $=86$ en pacientes.

Cuestionario para padres

Escala de evaluación

Cuestionario

Cuestionario Los coeficientes de confiabilidad (Spearman-Brown) varían de aceptable (.77) a excelente (.99).

La consistencia interna fue obtenida con un alfa de 0.87 . En cuanto a la validez de constructo muestra una correlación positiva muy alta de .77 con la Escala de Tendencia Empática Emocional de 1972.

Coeficiente de Alpha sobre .90

El alfa de Cronbach se calculó para el EQ en su conjunto utilizando a todos los sujetos de los estudios 1 y 2 . El Alfa obtenido fue de 0,92 , que es alto.

Instrumentos citados en: IRI: Gibson et al, 2014; Lavoie et al, 2014; Sparks et al, 2010; Matsumoto et al, 2015; McDonald, 2017; Martín, M.C. et al, 2017.; Gagen, E. et al, 2017. OSCARS: Roberts et al, 2014. SRS: Olszewski, A. et al, 2017. CELF-5: McDonald, 2017, BEES: McDonald, 2017, EQ: McDonald, 2017.

\section{2.- Descripción de los instrumentos de la Tabla $N^{\circ} 2$.}

1.- The Interpersonal Reactivity Index, IRI. (Davis, 1983). Escala Likert (0 no me describe bien a 4 me describe muy bien), con las siguientes dimensiones: de toma de perspectiva, malestar personal, fantasía, y preocupación empática general en los individuos. Escala validada en Chile por Fernández, Dufey \& Kramp, 2011.

2.-Observable Social Cognition A Rating Scale, OSCARS. (Healey et al, 2015). Instrumento que evalúa la manifestación del comportamiento cognitivo en el mundo real. Escala de calificación, de papel y lápiz, que incluye una escala de informantes completada por un miembro de la familia, amigo cercano o cuidador, y una escala de entrevistador que refleja impresión general del entrevistador basada en el informe del informante. Presenta 8 dominios que se evalúan en escalas de 7 puntos Likert, desde 1 sin evidencia de dificultad hasta 7 evidencia extrema de dificultad. Los dominios son: reconocimiento de emociones, sesgo atributivo hostil, saltar a conclusiones, flexibilidad cognitiva social, juicios, comprensión de sutileza en conversaciones, perspectiva y conocimiento social. Las puntuaciones se suman en los ocho dominios para obtener una puntuación total que oscila entre 8 y 56, con puntuaciones más altas indicando una mayor disfunción.

3.- Social Responsiveness Scale SRS" (Constantino et al., 2003) Escala en formato de cuestionario para padres que mide diferentes dimensiones del comportamiento interpersonal y conductas repetitivas y estereotipadas propias del espectro autista. Tiene 65 ítems.

4.- . Clinical Evaluation of Language Fundamentals (CELF-5) (Wiig et al, 2013). Test que evalúa inferencia conversacional y pragmática en sujetos desde la niñez hasta la adultez temprana. 
5.- Balanced Emotional Empathy Scale (BEES) (Mehrabian, 1996). Cuestionario de autoreporte que evalùa en forma unidimensional la empatía afectiva o emocional. Consta de 30 items, 15 de los cuales están redactados positivamente, y los otros negativamente.

6.- Empathy Quotient (EQ) (Baron-Cohen et al, 2004). Cuestionario de autoreporte de empatía para adultos con inteligencia dentro del promedio.

c. Sub dominio: Conocimiento Social

También denominada Esquemas Sociales, vinculada con la conciencia de los roles, normas y objetivos que caracterizan las situaciones sociales y guían las interacciones sociales. Implica el conocimiento sobre lo que es típico en situaciones sociales específicas.

\section{1.- Instrumentos de evaluación, subdominio Conocimiento Social}

Tabla 3.Instrumentos de evaluación de la cognición social. Subdominio Conocimiento Social

\begin{tabular}{lll}
\hline TEST Y AUTOR & FORMATO & ASPECTOS PSICOMÉTRICOS \\
\hline 1.- The Social Functioning Scale (SFS) & Cuestionario & Versión abreviada: Alfa Cronbach 0.76, Co- \\
(Birchwood et al., 1990) & & eficiente de correlación de spearman: 0.42. \\
& & Área bajo la curva ROC: 0.73 \\
& & Adaptación al español
\end{tabular}

2.- The Social Knowledge test (Achim et al, 2012b)

3.- Marlowe-Crowne Social Desirability Scale MCSDS

(Crowne \& Marlowe, 1960)

4.- Escala de Deseabilidad Social de Crowne y Marlowe (EDSCM)

5.- Social Skill Performance Assessment (SSPA) Escala de evaluación (Patterson et al., 2001)

6.-The Role Functioning Scale (RFS) (Goodman Escala de evaluación Coeficiente de correlación 0,80 et al., 1993). y respuesta

Escala de evaluación

Validez convergente: $r=0.44, p b 0.001$, Confiabilidad interna excelente: $\mathrm{r}=0.97, \mathrm{pb} 0.001$ )

Confiabilidad de la EDSCM para la muestra de estudiantes fue de $\alpha=76$, alpha de Cronbach, reportados por investigaciones recientes, estadounidenses y canadienses: de. 60 a .75 , española $=.78$

Adaptación argentina

Confiabilidad ICC $<0.70$

Instrumentos citados en: SFS: Brown et al, 2014; Tas et al, 2012; Maat et al, 2014; Sung-Wan, K. 2017; Biagianti et al, 2017. The Social Knowledge test: Lavoie et al, 2014. MCSDS: Renard et al, 2012. Assessment of Interpersonal Problem Solving Skills: Thaler et al, 2014.SSPA: Roberts et al, 2014; Kalin et al, 2015. Functional Outcome Measures: Wynn et al, 2010. MASC: Dickinson et al, 2007.

\section{2.- Descripción de los Instrumentos de la Tabla $N^{\circ} 3$}

1.- The Social Functioning Scale, SFS. (Birchwood et al, 1990). Cuestionario de 79 ítems administrado por un entrevistador. Busca evaluar aspectos del funcionamiento social en el mundo real y en comunidad. Tiene 7 dimensiones del funcionamiento social: compromiso social/retraimiento, comunicación interpersonal, independencia/ desempeño, recreación, actividades pro-sociales, independencia/competencia, y empleo. Los ítems son calificados en una escala de 4 puntos para frecuencia o habilidad y los puntajes más altos indican mayor competencia. 2.-The Social Knowledge Test. (Achim et al, 2012). Consiste en la presentación de situaciones hipotéticas originalmente inspiradas en temas sociales en listados en Blair y Cipolotti (2000). Se pregunta a los participantes respecto de cómo la gente en general se sentiría en 14 situaciones o ítems que componen la prueba. Una vez que el examinador lee la situación los participantes entregan sus respuestas las cuales son puntuadas con 1 o 0 de acuerdo a una tabla de corrección pre determinada. 
3.- Marlowe-Crowne Social Desirability Scale, MCSDS.(Crowne \& Marlowe, 1960). Escala de auto informe que evalúa la tendencia de las personas a reportar experiencias con el fin de comportarse de una manera culturalmente deseable. Tiene 33 enunciados y los participantes deben decir si aplican o no a ellos mismos.

4.- Adaptación argentina de la escala MCSDS. Evalúa deseabilidad social independientemente de psicopatología y consta de 33 ítems que se contestan por elección forzada entre Verdadero y Falso. A mayor puntaje corresponde mayor deseabilidad social. En algunos ítems a cada respuesta verdadera le corresponde el puntaje 1 y a cada respuesta falsa le corresponde el puntaje 0 . El resto de los ítems se puntúa de forma inversa. El puntaje total se obtiene con la suma de los puntajes de cada ítem.

5.- Social Skill Performance Assessment, SSPA. (Patterson et al, 2001). Consiste en juegos de roles de interacción de 3 minutos sobre temas predeterminados con un investigador asociado calificado. Las conversaciones son grabadas y codificadas por evaluadores ciegos, calificados para la fiabilidad. Para cada escena, se evalúan los siguientes dominios: Habilidad paralingüística (la suma de los dominios de fluidez y claridad), Propiedad social, Habilidad de conversación (valorado sólo para la escena 1), y Emociones. Los puntajes de los dominios son sumados a lo largo de las escenas para llegar a un puntaje total que va de 9 a 45 .

6.- The Role Functioning Scale, RFS. (Goodman et al, 1993). Escala de evaluación de resultados funcionales: habilidades para la vida independiente, funcionamiento social y funcionamiento laboral, de 30 minutos de duración aproximada. La escala se puntúa desde 1 (severamente dañado) a 7 (máximo funcionamiento) e incluye puntajes ancla que permiten detallar la cantidad y calidad del funcionamiento.

\section{d. Sub dominio: Estilo de atribución}

Se refiere a cómo uno explica las causas de los resultados positivos y negativos en su vida. La mayoría de los estudios en esta área se han centrado en pacientes con paranoia o ideas delirantes de persecución. Por lo mismo, se ha visto que podría estar estrechamente relacionado con el mecanismo psicológico de la ilusión persecutoria.

\section{1.-Instrumentos de evaluación subdominio Estilo de Atribución.}

Tabla 4. Instrumentos de evaluación de la cognición social. Subdominio Estilos de Atribución

\begin{tabular}{|c|c|c|}
\hline TEST Y AUTOR & FORMATO & ASPECTOS PSICOMÉTRICOS \\
\hline $\begin{array}{l}\text { 1.-Attributional Style Questionnaire } \\
\text { (ASQ) } \\
\text { (Peterson et al., 1982) }\end{array}$ & Cuestionario & $\begin{array}{l}\text { Consistencia interna: cronbach } 75 \text { y } 72 \text {. Consistencia } \\
\text { entre áreas: } 23 \text { a } 59\end{array}$ \\
\hline $\begin{array}{l}\text { 2.-The Ambiguous Intentions Hostility } \\
\text { Questionnaire (AIHQ) } \\
\text { (Combs et al., 2007) }\end{array}$ & Cuestionario & Confiabilidad $=6.27 \pm 1.27 ;$ valide $z=2.73 \pm 1.19$ \\
\hline $\begin{array}{l}\text { 3.- The Social Attributions Test- Mul- } \\
\text { tiple Choice (SAT-MC) } \\
\text { (Bell et al., 2010) }\end{array}$ & Escala de evaluación & $\begin{array}{l}\text { Confiabilidad con coeficiente Spearman-Brown }= \\
0.75\end{array}$ \\
\hline $\begin{array}{l}\text { 4.- Internal, Personal and Situational } \\
\text { Attribution Questionnaire (IPSAQ) } \\
\text { (Kinderman \& Bentall, 1996) }\end{array}$ & Cuestionario & $\begin{array}{l}\text { Alpha de cronbach de } 0.71 \text { en pacientes con EQZ a } \\
0.87 \text { voluntarios sanos }\end{array}$ \\
\hline $\begin{array}{l}\text { 5. "Health Action Process Approach } \\
\text { Inventory" (HAPA) (Schwarzer, 2008) }\end{array}$ & $\begin{array}{l}\text { Escala tipo likert y } \\
\text { acelerómetro para } \\
\text { medir actividad física }\end{array}$ & $\begin{array}{l}\text { La consistencia interna para } 7 \text { de las } 11 \text { subescalas } \\
\text { utilizadas en el estudio varió entre } 0.72 \text { y } 0.95 \text {. }\end{array}$ \\
\hline \multicolumn{3}{|c|}{$\begin{array}{l}\text { Instrumentos citados en: ASQ: Wang et al, 2013. AIHQ: Gibson et al, 2014; Roberts et al, 2014; Vázquez-Campo et } \\
\text { al, 2016; Rocha \& Queirós, 2013; Kalin et al, 2015; Combs et al, 2007; Roberts \&Penn, 2009. The agency task: Schi- } \\
\text { mansky et al, 2010. SAT-MC: Minor et al, 2015; Minor \& Lysaker, 2014. IPSAQ: Tas et al, 2012; Mehta et al., 2014; } \\
\text { Brown et al, 2014; Fernandez-Gonzalo et al. 2015; Savla et al, 2012; Donohoe et al, 2012; Tas et al, 2013. HAPA: Ar- } \\
\text { bour-Nicitopoulos et al, 2017. }\end{array}$} \\
\hline
\end{tabular}




\section{2.-Descripción de los instrumentos de la Tabla $N^{\circ} 4$}

1.-Attributional Style Questionnaire, ASQ. (Peterson et al, 1982). Cuestionario que valora tres de las dimensiones del estilo de atribución: interno, estable y global. Se miden 36 elementos de respuesta individual, de lápiz y papel, donde se presentan 12 escenarios hipotéticos (6 positivos y 6 negativos). Se anima a los participantes a imaginar la situación que ocurre y a proponer una solución. La situación se valora en cada una de las 3 dimensiones internas-externas (Lugar), estabilidad-inestabilidad (Estabilidad), globalidad-especificidad (Globalidad), e importancia para uno mismo.

2.- The Ambiguous Intentions Hostility Questionnaire, AIHQ. (Combs et al, 2007). Los participantes escuchan cinco historias en segunda persona, presentadas oralmente, que describen interacciones sociales con un resultado negativo. Después de cada historia, los participantes entregan tanto opiniones de respuesta libre como de tipo Likert, sobre si el otro personaje guardaba una intención hostil hacia el participante, cuánto debe ser culpado el personaje por la situación, y cuán agresivamente el participante respondería. Es una manifestación del comportamiento en el mundo real.

3.- The Social Attributions Test- Multiple Choice, SAT-MC. (Bell et al, 2010). Es una medida no verbal de teoría de la mente. A los participantes se les presenta una animación abstracta de 1 minuto de duración, con situaciones sociales que incluyen un triángulo grande, un triángulo pequeño y un círculo pequeño. Después de ver la animación dos veces, a los sujetos se les realizan 19 preguntas de selección múltiple relacionadas a las interacciones en la animación. El SAT-MC ha mostrado propiedades psicométricas fuertes en los estudios de la esquizofrenia (Bell et al., 2010; Pinkham et al., 2014).

4.-Internal, Personal and Situational Attribution Questionnaire, IPSAQ. (Kinderman \& Bentall, 1996). Test de 32 ítems, que describen situaciones sociales positivas y negativas. Se solicita manifestar la causa más posible para la ocurrencia de un evento y indicar si la causa se debe a uno mismo (atribuciones internas), a otras personas (atribuciones personales) o a circunstancias (atribuciones situacionales). Se generan seis puntajes de sub-escalas, a través de la suma de los números de las atribuciones internas, personales y situacionales escogidas para los ítems positivos y negativos. Se obtienen dos puntajes de sesgo cognitivo. El puntaje del sesgo externalizador se calcula restando el número de atribuciones internas para eventos negativos del número de atribuciones internas para eventos positivos. El puntaje del sesgo de personalización (tendencia a atribuir eventos negativos a otros en vez de a factores situacionales), se calcula dividiendo el número de atribuciones personales para eventos negativos en la suma de las atribuciones tanto personales como situacionales para eventos negativos.

5.- Health Action Process Approach Inventory" (HAPA) (Schwarzer, 2008). Inventario con 11 subescalas para adultos con esquizofrenia. Consta de dos etapas: $1^{\circ}$ motivación preintencional, donde evalúa creencias relacionadas a percepción de riesgos, expectativa de resultados y confianza para desempeñar la meta de conducta. $2^{\mathrm{a}}$ etapa, la fase de volición post intencional, se evalúan creencias auto-regulatorias de planificación, iniciación y mantenimiento de la conducta.

\section{e. Sub dimensión: Teoría de la Mente. ToM}

También conocido como mentalización o atribución del estado mental. Capacidad de representar los estados mentales propios y de otras personas en términos de pensar, creer o pretender. Se asocia con la capacidad del individuo para entender falsas creencias, sugerencias, intenciones, humor, los engaños, la metáfora y la ironía. La mayoría de los estudios han puesto de manifiesto las deficiencias de la teoría de la mente en pacientes con esquizofrenia. 
1.- Instrumentos de evaluación, subdominio Teoría de la Mente.

Tabla 5. Instrumentos de evaluación. Subdominio ToM

\begin{tabular}{|c|c|c|}
\hline TEST Y AUTOR & FORMATO & ASPECTOS PSICOMÉTRICOS \\
\hline $\begin{array}{l}\text { 1.- The Reading the Mind in the Eyes } \\
\text { Test (RMET) } \\
\text { (Baron-Cohen et al., 2001) }\end{array}$ & Imágenes & $\begin{array}{l}\text { Consistencia interna, Alpha de cronbach: } 60,5 \text {, Con- } \\
\text { fiabilidad interna: } 71,9 \text { Fiabilidad test re test: } 83,3 \\
(95 \%=74,5 \text { a } 90,2) \text {. } \\
\text { Adaptación Sueca, japonesa, italiana, }\end{array}$ \\
\hline $\begin{array}{l}\text { 2.- The Awareness of Social Inference } \\
\text { Test, (TASIT) } \\
\text { (McDonald et al., 2003) }\end{array}$ & Video & $\begin{array}{l}\text { Alpha de cronbach, para escala Likert }=0.92 \text {. } \\
\text { Validación Dinamarca }\end{array}$ \\
\hline $\begin{array}{l}\text { 3.- Social Cognition and Object Rela- } \\
\text { tions Scale (SCORS) } \\
\text { (Westen et al., 1991) }\end{array}$ & Escala de evaluación & Confiabilidad excelente, rangos de 0.83 a 0.93 \\
\hline $\begin{array}{l}\text { 4.- Recognition of Faux Pas } \\
\text { (Baron-Cohen, 1997) }\end{array}$ & Preguntas y respuestas & confiabilidad $=7.00 \pm 2.16 ;$ validez $=3.73 \pm 1.42$ \\
\hline $\begin{array}{l}\text { 5.- The Movie for the Assessment of } \\
\text { Social Cognition (MASC) } \\
\text { (Dziobek et al., 2006) }\end{array}$ & Video & $\begin{array}{l}\text { Confiabilidad }=0.501, \mathrm{P}<0.001 \\
\text { po0 }\left(\mathrm{r}^{1 / 40.501,}\right. \\
\text { Confiabilidad }=0.501, \mathrm{p}<0.001\end{array}$ \\
\hline 6-Faux-Pas Task (Stone et al., 1998). & $\begin{array}{l}\text { Historias con preguntas } \\
\text { posteriores }\end{array}$ & El Alfa de Cronbach.62 \\
\hline $\begin{array}{l}\text { 7.Beck Cognitive Insight Scale } \\
\text { (BCIS; Beck et al,, 2004) }\end{array}$ & Escala de autoinforme. & $\begin{array}{l}\text { La consistencia interna es suficiente para fines de } \\
\text { investigación con } \alpha \text { de Cronbach }=0.687 \text {. }\end{array}$ \\
\hline $\begin{array}{l}\text { 8.- Movie for the Assessment of So- } \\
\text { cial Cognition" MASC (Dziobek et } \\
\text { al., 2006) }\end{array}$ & Video & $\begin{array}{l}\text { Presenta consistencia interna evaluada mediante un } \\
\text { alpha de } 0.84 \text { para el total de la escala. }\end{array}$ \\
\hline \multicolumn{3}{|c|}{$\begin{array}{l}\text { Instrumentos citados en: RMET: Brown et al, 2014; Fernandez-Gonzalo et al, 2015., Gibson et al, 2014; Guastella et } \\
\text { al, 2015; Savla et al, 2012; Buck et al, 2015; Tso et al 2010; Tas et al, 2012; Martínez-Domínguez et al, 2015; Balogh } \\
\text { et al, 2014; Woolley et al, 2014; Schimansky et al, 2010; Thaler et al, 2014; Wang et al, 2013; Lysaker et al, 2013b; } \\
\text { Lugnegård et al, 2013; Donohoe et al, 2012; Browne et al, 2016; Robertson et al, 2013; Whitton\& Henry, 2013; Tas et } \\
\text { al, 2013; Köther, U. et Al, 2017.; Gagen, E. et Al, 2017.; Okruszek et al, 2017; EŞSizOĞLU et al, 2017; Charernboon } \\
\text { \& Patumanond, 2017. TASIT: Davis et al, 2013; Wynn et al, 2010; Woolley et al, 2014; Sparks et al, 2010; Kalin et al, } \\
\text { 2015; Horan et al, 2012; Browne et al, 2016; Rassovsky et al, 2015; McDonald, 2017; Mervis et al, 2017; Palumbo et } \\
\text { al, 2017. SCORS: Lysaker et al, 2010. Recognition of Faux Pas: Vázquez-Campo et al, 2016. Faux-Pas Task: Mehta } \\
\text { et al, 2014; Hasson-Ohayon et al, 2015. Köther et Al, } 2017 \text { MASC: Andreou et al, 2015; Kocsis-Bogár et al, 2017.. }\end{array}$} \\
\hline
\end{tabular}

\section{2.- Descripción de los Instrumentos de la Tabla $N^{\circ} 5$}

1.- The Reading the Mind in the Eyes Test, RMET. (Baron-Cohen et al, 2001). Este test es una tarea que comprende 36 fotografías y examina la dimensión social y de percepción de la ToM. Los participantes escogen la mejor palabra, de un total de cuatro, lo que mejor describa a una persona (piensa o siente) solo con mirar sus ojos. Estudios recientes verificaron la fiabilidad de esta herramienta midiendo habilidades ToM en la esquizofrenia (Bora \& Pantelis, 2013), un estudio sueco aseguró la fiabilidad test-retest para la versión de niños del RMET, no encontrando indicios de efectos aprendizaje en el caso de ser repetido el test (Hallerback et al., 2009).

2.- The Awareness of Social Inference Test, TASIT. (McDonald et al, 2003). Consiste en videos de 16 a 60 segundos, donde actores representan escenas que incluyen mentiras y sarcasmos. Durante la visualización se entrega información al espectador respecto de la naturaleza de la conversación entre los actores. Los participantes deben usar esa información para inferir pensamiento, intenciones, sentimiento y creencias de los actores y responder preguntas de elección forzada en que se pregunta: 1) Qué están haciendo los personajes, 2) qué están tratando de decir entre ellos, 3) Qué están pensando y 4) Qué están sintiendo. Este test entrega un puntaje total (máximo 64) y puntajes de sub escala para escenas de mentiras y sarcasmo (máximo 32 puntos cada una). 
3.- Social Cognition and Object Relations Scale, SCORS. (Westen et al., 1991). Método para determinar, a través de las respuestas del Test de Apercepción Temática, TAT (Murray, 1943), hasta qué punto las personas aprecian la complejidad de las experiencias subjetivas, causalidad social, y las necesidades de participantes múltiples en intercambios sociales. Contiene cuatro escalas que van de ' 1 ' (representaciones menos maduras) a ' 5 ' (representaciones más maduras). Esas escalas son: Complejidad de Representaciones (habilidad de diferenciar entre las perspectivas del mismo y de otros, y de reconocer la complejidad de los motivos y experiencias subjetivas de otros), tono de los sentimientos (hasta qué punto se espera que las interacciones interpersonales sean gratificantes versus emocionalmente dolorosas), Capacidad para la inversión emocional en Relaciones y Estándares Morales (actitudes hacia otros como objetos de una conexión comprometida, más que usados para su propia satisfacción) y el Entendimiento de la Causalidad Social (habilidad para formular ideas lógicas, creíbles, conscientes psicológicamente, y complejas sobre lo que guía el comportamiento). SCORS mide las representaciones del mundo social, en lugar de representaciones obtenidas y filtradas por el auto-informe.

4.- Recognition of Faux Pas (Baron-Cohen, 1997). Presenta 10 escenarios en los que uno de los personajes comete o no una situación de metida de pata (faux pas). Se pide a los participantes que identifiquen si el personaje dijo algo que debió decir y explicar el porqué. Los puntajes se generan en relación con la correcta detección y explicación de la situación de metida de pata. Un desempeño bajo indica una sensibilidad reducida en relación a los pensamientos y sentimientos de los demás, como también atribuciones inadecuadas sobre trasgresiones e insultos de otros.

5.- The Movie for the Assessment of Social Cognition, MASC. (Dziobek et al, 2006). Película de 15 minutos donde 4 personas cenan juntas. Los personajes presentan rasgos estables diferentes (sociable, tímido, egoísta) y en el transcurso de la película experimentan varias situaciones, provocando distintas emociones y estados mentales (rabia, celos, repulsión). El nivel de intimidad entre los personajes varía a medida que son extraños o amigos. Consecuentemente cada personaje desarrolla su propia dinámica hacia los otros. A los participantes se les pide que traten de entender los estados mentales de los personajes. En determinados momentos de la película, se realizan preguntas de opción múltiple respecto a sus sentimientos (15 ítems), pensamientos (14 ítems) e intenciones (4 ítems), en pausas dadas. Seis preguntas controladas se incluyen para permitir la evaluación de la capacidad de inferencia no social (ej.: ¿cómo estuvo la temperatura esta tarde?, las respuestas tienen que ser inferidas por la ropa de los protagonistas). MASC se ha usado para evaluar la cognición social en el autismo, esquizofrenia, depresión y desorden de personalidad, y ha recibido validación clínica.

6.- Faux-Pas Task (Stone et al, 1998). Consiste en 10 historias, en las cuales ha ocurrido una "metedura de pata", y 10 historias control. La "metedura de pata" ocurre cuando el hablante dice algo sin considerar que el oyente tal vez no quiera escucharlo o bien porque puede herir sus sentimientos. Después de cada historia a los participantes se les realizan seis preguntas sobre el reconocimiento de la "metedura de pata" (entendiendo el estado mental del hablante y el oyente, y entendiendo el estado emocional del oyente). La tarea evalúa las atribuciones emocionales y cognitivas y el puntaje para cada historia va entre 0 y 7 (rango de la tarea: 0-70).

7.- Beck Cognitive Insight Scale" (Beck et al, 2004). Escala de dos dimensiones: autorreflexión y autoconfianzase combinan estas dos dimensiones en un "Índice compuesto" (autorreflexión menos autoconfianza) que debe reflejar el nivel de introspección del paciente restringido por la cantidad de certeza sobre sus creencias.

8.- Movie for the Assessment of Social Cognition MASC (Dziobek et al., 2006). Prueba audiovisual que evalúa dificultades sutiles en Teoría de la Mente. Consta de película de 15 minutos sobre dos hombres y dos mujeres organizando y pasando una velada nocturna. Se le pide al participante que respondan 45 preguntas de opción múltiple relacionadas con los sentimientos, pensamientos e intenciones. 


\section{f. Instrumentos que integran varios subdominios de la Cognición Social.}

\section{1.- Instrumentos de evaluación de la cognición social, que integran varios subdominios.}

Tabla 6. Instrumentos de evaluación de la cognición social que integran varios subdominios

\begin{tabular}{lll}
\hline TEST Y AUTOR & FORMATO & ASPECTOS PSICOMÉTRICOS \\
\hline 1.- The Hinting Task & Cuestionario & Alfa de Cronbach fue de 0,69. la fiabilidad test-retest, así \\
& & como los valores de la consistencia interna es superior a \\
(Corcoran et al., 2005) & 0,80 para todas las historias, y la fiabilidad test-retest fue \\
& igual o mayor a 0,70 Validación USA, Inglaterra, España
\end{tabular}

2.- Escala de cognición social (ECS)

(Fuentes et al., 2011)

3.- Functional Independence Measure V.3.0 (Keith, R. et al., 1987).

4.- MATRICS Consensus Cognitive Battery (MCCB) (Nuechterlein et al., 2008)
Escala de evaluación

Fiabilidad: 70

Validación española
La validez y fiabilidad han sido vastamente investigadas ya que la FIM se utiliza como una medida estándar en los sistemas de salud de Norte América.

Fiabilidad test-re test.

Instrumentoscitados en: The Hinting Task: Fiszdon et al, 2007; Brown et al, 2014; Roberts et al, 2014; Minor et al, 2015; Lysaker et al, 2013; Buck et al, 2015; Vázquez-Campo et al, 2016; Tas et al, 2012; Martínez-Domínguez et al, 2015; Fanning et al, 2012; Rocha \&Queirós, 2013; Minor\&Lysaker, 2014; Bell et al, 2009; Park \& Kim, 2014; Kalin et al, 2015; Thaler et al, 2014; Lysaker et al, 2013b; Bliksted et al, 2014; Maat et al, 2012; Horton\&Silverstein, 2008; Donohoe et al, 2012; Browne et al, 2016; Robertson et al, 2013. ECS:Álvarez et al, 2013; Rodríguez et al 2013; Fuentes et al, 2011. Functional Independence Measure: Bagge, E. et Al, 2017MCCB: Bo et al, 2017.; Zhang, B. et Al, 2017.

\section{2.- Descripción de los Instrumentos de la Tabla $N^{\circ} 6$}

1.- The Hinting Task (Corcoran et al, 2005). Esta tarea evalúa la habilidad para inferir las intenciones que hay detrás del discurso. Comprende 10 historias cortas que implican la interacción entre dos personajes. Cada historia termina con uno de los personajes "soltando una indirecta" y el participante tiene que explicar aquello que intenta comunicar el personaje. Cada historia es leída por el experimentador dos veces para compensar posibles daños en la memoria de trabajo de la persona evaluada.

2.- Escala de cognición social, ECS. (Fuentes et al, 2011). Este instrumento tiene como finalidad medir diferentes componentes de la cognición social de manera integrada: identificación de estímulos, percepción emocional y percepción social. Tiene un primer ítem que corresponde a una pregunta abierta en la que el entrevistado debe enumerar detalles, elementos y objetos que aparecen en fotografías en un minuto y medio. El Ítem 2 aborda las emociones y a las personas al observar imágenes y en el 3 se pide al participante que indique verdadero y falso, ante una serie de frases relativas a lo que sucede en las imágenes.

3. Functional Independence Measure V.3.0 (Keith et al., 1987). Escala de observación Consta de 18 dominios de comportamiento, 13 de dominio motor y 5 de dominio cognitivo social, para medir actividades y limitaciones de actividad en la vida diaria, que refleja lo que

4.- MATRICS Consensus Cognitive Battery” (MCCB) (Nuechterlein et al., 2008). Evalúa deterioro cognitivo en la esquizofrenia. La función cognitiva es medida a través de 7 dominios: velocidad de procesamiento, atención/vigilancia, memoria de trabajo, aprendizaje verbal, aprendizaje visual, razonamiento y resolución de problemas y cognición social. 


\section{g. Programas de entrenamiento de la Cognición Social.}

\section{1.- Programas de rehabilitación de la CS}

Tabla 7. Intervenciones para el entrenamiento de la cognición social

\begin{tabular}{|c|c|c|c|c|}
\hline Nombre y Autor & Subdominio & Formato & Duración & Idioma \\
\hline $\begin{array}{l}\text { 1. Mind Reading, inter- } \\
\text { active Guide to Emotions } \\
\text { (MRIGE). }\end{array}$ & $\begin{array}{l}\text { Reconocimiento } \\
\text { emocional facial }\end{array}$ & Computacional individual & $\begin{array}{l}12 \text { semanas, } 1 \text { vez por } \\
\text { semana, } 1 \text { hora por } \\
\text { sesión }\end{array}$ & Inglés \\
\hline
\end{tabular}

(Lindenmayer et al., 2013)

2.Cognitive-Emotional Rehabilitation (REC)

(Vendittelli et a.1, 2003)

3.Family-assisted Social Cognition and Interaction Training (F-SCIT)

(Roberts \& Penn, 2009.)

4.Social stimulation (SS)

(Lysaker et al., 2009)

5.Social-Cognition-and-Interaction-Training (SCIT)

(Roberts, et al., 2006)

6.Metacognitive-and-social-cognition-training (MSCT)

(Hogarty et al., 2004)

\section{NeuroPersonalTrainer (NPT-MH)}

Instituto Guttmann, (Barcelona, España) 8. Micro-Expression Train-
ing Tool (METT)

(Russell et al., 2008)

9. Metacognitive Training for patients with Schizophrenia" (MCT). (Moritz \& Woodward, 2007;

Köther et al., 2017)

10. Computerised Interactive Remediation of Cognition - a Training for Schizophrenia" (CIRCuiTS) (Reeder et al., 2017)
Percepción emo- Grupal, uso de manual estruccional, interacción turado social

Teoría de la mente. Estilos atribucionales.

Grupal, uso de manual, uso de videos

Interacción social

Percepción emocional, estilo atribucional, teoría de la mente.

Sesgos cognitivos, Grupal, uso de manual descarTeoría de la mente, reconocimiento de emociones

Percepción emocional, interacción social, estilos atribucionales, teoría de la mente.

Entrenamiento en micro expresión facial

Teoría de la Mente

Teoría de la mente internet, administrada por un terapeuta, pero suplementada con sesiones independientes para facilitar la práctica.

Semi estructurado con actividades sociales variadas

Grupal, no indica manual

Computacional individual o grupal (6 personas), programa nerocognitivo con un módulo de Cognición Social.

computacional individual como grupos abiertos donde el contenido es presentado a través de diapositivas Power Point con ejercicios y entrega de información.
Seis meses, 1 vez por semana, 75-90 min por sesión

14 sesiones 1 vez por semana, 80 minutos por sesión.

Inglés

Sesiones grupales configuradas

4 sesiones, 1 vez a la semana, medio día (4 hrs.)

20 a 24 sesiones, 1 vez a la semana, 60 minutos de duración.

18 sesiones, 1 vez por semana.

Inglés, Alemán, Portugués Coreano, Polaco, Ruso

Sesiones de 1 hora, dos veces a la semana durante 4 a 5 meses, mínimo de 15 horas.

Inglés, español.

12 sesiones, una hora Inglés por semana

Ocho sesiones (dos en- Inglés, focadas en cognición Alemán social).

40 sesiones de hasta Inglés. una hora, al menos 3 veces a la semana. Además de la posibilidad de realizar sesiones independientes. 


\section{2.- Descripción de los programas de la Tabla $N^{\circ} 7$}

1.- Mind Reading, interactive Guide to Emotions, MRIGE. (Lindenmayer et al, 2013). Este software fue desarrollado para mejorar las habilidades de reconocimiento emocional facial en personas con Asperger. De manera adicional se ha encontrado que es también útil para la práctica individualizada de personas con esquizofrenia. (Lindenmayer et al., 2013). Consiste en un sistema taxonómico de 412 emociones y estados mentales, agrupados en 24 grupos de emociones y 6 niveles de desarrollo del ciclo vital (desde los 4 años a la adultez). Cada emoción es definida y demostrada en 6 videos mudos de rostros, 6 audios y 6 ejemplos escritos de una situación que evoca tal emoción. La base de datos del MRIGE posee un panel de interacción que utiliza 3 aplicaciones específicas: 1) Biblioteca emocional, la que permite al usuario navegar libremente por entre los diferentes grupos de emociones, ver videos, escuchar audios y leer historias para comparar y practicar el reconocimiento de emociones. 2) En centro de aprendizaje, que tiene lecciones, cuestionarios y varios tareas desarrolladas para el entrenamiento recompensado. 3) La zona de juego, la cual se compone de 5 juegos educacionales que permiten al usuario jugar y estudiar las emociones al mismo tiempo. El uso de esta herramienta debe ser guiado por un profesional. 2. Cognitive-Emotional Rehabilitation, REC. (Vendittelli et al, 2003). Es un tratamiento grupal estructurado de seis meses, con co-terapeuta y uso de manual, cuenta con reuniones semanales de entre 75 a 90 minutos, participan entre 8 a 12 personas. Se basa principalmente en psico-educación con una aproximación de estilo cognitivo conductual. Las sesiones están orientadas a enseñar a los participantes a reconocer eventos y situaciones en las que estarían expuestos en la cotidianeidad. Está orientado a generar mejoras en la comunicación interpersonal y la adquisición de habilidades en resolución estructurada de problemas por medio de entrenamiento práctico y trabajo en el hogar. El tratamiento consta de tres fases, la primera tiene relación con el compartir objetivos de trabajo (motivación y consentimiento), aprender a estar atentos a emociones faciales (miedo, alegría, enojo, culpa, tristeza, vergüenza, celos, envidia, interés, desagrado y vergüenza), y entender el comportamiento de los demás. La segunda fase se denomina "navegador emocional" en el cual la actividad está enfocada al entrenamiento en reconocimiento de emociones y observación del comportamiento para entender los estados mentales de otros. En la tercera fase se recolecta la información del trabajo emocional realizado y se aborda desde cinco áreas: Contexto/situación, ánimo/emociones sentidas, pensamientos/imágenes de discapacidad mental, cuerpo/ sensaciones, acciones/comportamiento/actitudes/expresiones.

3. Family-assisted Social Cognition and Interaction Training, F-SCIT. (Roberts \& Penn, 2009). Extensión del SCIT. El F-SCIT. Fue diseñado como un programa de entrenamiento grupal de 14 sesiones, una por semana de 80 minutos con 15 minutos de descanso. Incluye las mismas tres fases de trabajo que el SCIT, pero con la modificación de que este programa está destinado a llevar a la práctica las habilidades entrenadas en la vida real. Para esto se agregó una fase individual en la etapa de Integración, ya que los participantes, a pesar de ser alentados a presentar y poner en discusión sus dificultades personales en el grupo, no expresan todos sus problemas de la vida real. Finalmente se asigna a un familiar o amigo cercano como "compañero de entrenamiento". 4.- Social Stimulation, SS. (Lysaker et al., 2009). Intervención en la cual se le asigna un acompañante voluntario entrenado en psicoterapia a un paciente estabilizado. La intervención consiste en sesiones de cuatro medios días de actividades semi-estructuradas desarrolladas cada tres semanas. Se incluyen conversaciones acerca de actividades cotidianas con el paciente, visita a un café, trabajos de pintura de cuadros en compañía del compañero voluntario y asistidos por un arte terapeuta, observar una comedia en un teatro local y finalmente una sesión de retroalimentación en la cual el voluntario con el paciente discuten sobre la forma de resolución de problemas cotidianos.

5. Social-Cognition-and-Interaction-Training, SCIT. (Roberts, et al., 2006).Intervención grupal con sesiones de 1 hora aproximada de duración, distribuidas en 20 a 24 semanas, dependiendo de la velocidad con la que el grupo trabaje en cada sesión. Cada grupo debe estar integrado por dos terapeutas y con 4 a 8 pacientes. SCIT utiliza una combinación de psico-educación, práctica de habilidades, juegos de estrategia, ensayo heurístico y asignaciones de tareas para remediar los déficits y disminuir los sesgos en la cognición social. Cada participante del grupo es alentado a identificar a un "compañero de práctica" con quien trabajar de manera adicional a las tareas asignadas. El tratamiento comprende tres fases: Emociones (percepción emocional disfuncional), Entendiendo situaciones (Estilos de sesgo atribucional, disfunción de la ToM), Integración (aplicación de habilidades aprendidas en su vida cotidiana). 
6.- Metacognitive-and-social-cognition-training, MSCT. (Hogarty et al, 2004). Comprende 18 sesiones, una sesión semanal en la semana 1 y 10; dos sesiones semanales de la semana 2 a la 9. En la primera sesión se trabaja sobre la relación entre emociones, pensamientos y comportamientos, enfocándose en los errores que pueden ocurrir en situaciones sociales. Desde la semana 2 a la 9 se inicia cada sesión con psicoeducación metacognitiva, para luego pasar a una práctica de remediación en interacción social, esto por medio de ejercicios para entrenar formas específicas de procesamiento de la información e ilustrar sesgos cognitivos en la vida cotidiana. En la sesión final, los participantes reportan la aplicación del programa en su vida diaria.

7.- NeuroPersonalTrainer, NPT- MH. Instituto Guttmann, (Barcelona, España). Corresponde a un software diseñado para personas en las primeras etapas de la psicosis. Las imágenes, videos e historias de esta intervención representan situaciones sociales comunes, en las que se ve a un adulto joven que puede encontrarse en diferentes situaciones en el trabajo, en un pub, con amigos y familiares; además, se muestran los problemas a los que una persona, en esta primera etapa de la enfermedad, se pueda enfrentar: mal entendidos sociales, situaciones de "faux-pas", manejo de emociones en diferentes contextos sociales. Este modulo permite trabajar sobre diferentes aspectos del procesamiento emocional, teoría de la mente y sesgos cognitivos mediante 43 tareas multimedia organizadas por niveles de dificultad variables. El programa también incluye un módulo de rehabilitación cognitiva que involucra atención, memoria, y funciones ejecutivas con diferentes niveles de complejidad. 8.- Micro-Expression Training Tool, METT. (Russell et al, 2008). Entrenamiento en micro expresiones, se inicia mostrando cuatro pares de emociones comúnmente confusas, en secuencias de videos a cámara lenta (enojo-desagrado, desprecio-felicidad, miedo-sorpresa, miedo-tristeza). Tiene una sesión de práctica que consiste en 28 ensayos en que los participantes etiquetan las micro-expresiones con retroalimentación sobre su desempeño. Las siguientes sesiones de entrenamiento presentan 4 pares de emociones confusas la primera, etiquetado de 14 rostros (feliz, enojado, triste, disgusto, miedo, sorpresa, desprecio) la segunda, y una prueba de coincidencia de emociones de 50 elementos (feliz, triste, enojado, disgusto, neutral), para finalizar.

9.- Metacognitive Training for patients with Schizophrenia (MCT). (Moritz \& Woodward, 2007). Diseñado para tratar tendencias o sesgos cognitivos propios de la psicosis. El objetivo del MCT es aumentar la conciencia de los pacientes sobre las posibles "trampas del pensamiento" que se sospecha están involucradas en la patogénesis de la ideación paranoica. Además de aumentar la conciencia y el conocimiento sobre estos sesgos, se les enseña a los pacientes distintas formas de aumentar sus habilidades de afrontamiento. Pertenece al subdominio Teoría de la Mente. Son 8 sesiones grupales configuradas como grupos abiertos donde el contenido es presentado a través de diapositivas Power Point con ejercicios y entrega de información. Hay dos sesiones enfocadas en cogniciòn social. (Inglés y Alemán)

10.- Computerised Interactive Remediation of Cognition - a Training for Schizophrenia (CIRCuiTS) (Reeder et al, 2010). Programa metacognitivo, computarizado de rehabilitación cognitiva, se enfoca en desarrollar conocimiento y regulación metacognitiva, o la capacidad de entender y manejar efectivamente los propios procesos cognitivos, con el fin de beneficiar a cognición y el funcionamiento social. Un terapeuta facilita el desarrollo de motivación, metacognición y estrategias, además de la generalización del aprendizaje para su transferencia a metas en el mundo real. Pertenece al subdominio Teoría de la mente. Es una Terapia computacional vía internet, administrada por un terapeuta, pero suplementada con sesiones independientes para facilitar la práctica. 40 sesiones de hasta una hora, al menos 3 veces a la semana. Además existe de la posibilidad de realizar sesiones independientes. Idioma inglés.

\section{Conclusiones}

El objetivo de este artículo es aportar a la rehabilitación del paciente con esquizofrenia a través de una revisión sistemática de instrumentos y programas específicos respecto de la cognición social. En lo que se refiere a instrumentos, se entrega una síntesis de contiene 37 herramientas evaluativas, con sus respectivas citas, en más de 195 publicaciones revisadas (184 en inglés y 11 en español). Los criterios de selección fueron fundamentalmente, que se señalaran indicadores psicométricos y que la utilización del instrumento estuviera condicionado a evaluar lo que se deseaba medir con fines diagnósticos y/o como evidencia del impacto de un tratamiento determinado. Una primera conclusión al respecto apunta -en términos generales y desde una perspectiva crítica- que en la mayor parte de la literatura analizada, no se colocan las propiedades psicométricas de los instrumentos, si éste 
fue validado en el contexto social donde se utiliza, tampoco su justificación. Se puede señalar además, que la fiabilidad es baja en casi todos los test reportados, dado que para ello se requiere de un valor mínimo aconsejado de 0.80 y que sobre 0.95 es desaconsejable; también que el índice de Kappa, mencionado en varios de ellos, sólo indica acuerdo inter jueces, no estabilidad de las puntuaciones en el test; que el test de Ekman señalado en el subdominio Procesamiento Emocional estaría en un valor aceptable. Se agrega, en términos generales, respecto de la validez de un instrumento, los mejores análisis psicométricos son los realizados con análisis factorial, coeficientes de correlación canónico cuadrados, o ecuaciones estructurales, lo cual no se refleja en los artículos analizados. Al respecto, Carretero-Dios \& Pérez C. (2007), realizaron hace más de una década, un interesante análisis señalando que a menudo en las publicaciones no se justifica la utilización de una determinada herramienta y que los criterios que habría que tener en cuenta para la utilización de un test son: delimitación conceptual del constructo objeto de evaluación; información respecto de la construcción y evaluación cualitativa de ítems; resultado del análisis estadístico de los ítems; evidencias empíricas de la estructura interna de la prueba; resultados de la estimación de la fiabilidad donde índices de fiabilidad alrededor de 0.70 serían adecuados en caso de investigaciones y si se trata de diagnósticos el valor mínimo debería situarse en torno a 0,80 ; y por último, evidencias externas de la validez de las puntuaciones del test. Se estima, a fin de avanzar de manera más certera en la rehabilitación del paciente, que se debería señalar explícitamente en las publicaciones, lo que mide el instrumento, índices de validez y fiabilidad y si está debidamente adaptado al contexto.

Se describen 10 programas de rehabilitación de la CS. Estas son intervenciones con utilización de videos, imágenes y/o software; son programas individuales o grupales, con una frecuencia mínima de una sesión por semana. Tienen en común el entrenamiento en el reconocimiento emocional, la comunicación social, la adquisición de habilidades para la resolución de problemas, la psico-educación, los ejercicios vinculados a actividades de la vida cotidiana, y que en todos se indican éxitos terapéuticos. No obstante, sería importante conocer indicadores de evanescencia.

Es necesario mencionar que Inchausti et al. (2017) señalan, que los déficit sociales de la esquizofrenia se relacionarían más con dificultades de comprensión e integración de estados mentales propios, ajenos a situaciones interpersonales y/o déficit neuro-cognitivos aislados.

Estos autores abren con sus publicaciones, una nueva línea investigativa complementaria respecto de la rehabilitación social del paciente con esquizofrenia, en la cual habría que profundizar.

Finalmente, se concluye y reitera en la necesidad de explicitar, en la literatura científica, las características psicométricas de los instrumentos utilizados, como también la descripción de los programas de rehabilitación psico-social e índices de evanescencia, en beneficio de la rehabilitación, reinserción social y funcionalidad diaria del paciente con esquizofrenia.

\section{Referencias}

Achim, A. M., Ouellet, R., Roy, M. A., \& Jackson, P. L. (2012). Mentalizing in first-episode psychosis. Psychiatry Research, 196, $207-213$.

Álvarez, J. C., Tourinõ, R., Abelleira, C., Fernańdez, J., Baena, E., Giráldez, A., \& Bordón, R. (2013). Social cognition and schizophrenia: Differences between users of psychosocial rehabilitation day center and from a supported employment program. [Cognición social y esquizofrenia: Diferencias entre usuarios de centros de día de rehabilitación psicosocial y de un programa de empleo con apoyo] Rehabilitacion Psicosocial, 10, 4-9.

Andreou, C., Kelm, L., Bierbrodt, J., Braun, V., Lipp M., Yassari, A., \& Moritz, S. (2015) Factors contributing to social cognition impairment in borderline personality disorder and schizophrenia. Psychiatry Research, 229, 872-879.

Blair, R., \& Cipolotti, L. (2000). Impaired social response reversal: A case of 'acquired sociopathy'. Brain, 123, 1122-1141. doi: 10.1093/ brain/123.6.1122

Balogh, N., Égerházi, A., Berecz R., \& Csukly, G.(2014) Investigating the state-like and trait-like characters of social cognition in schizophrenia: A short term follow-up study. Schizophr Research, 159, 499-505.

Baron-Cohen, S., (1997). Hey! It was just a joke! Understanding propositions and propositional attitudes by normally developing children and children with autism. Isr. J. Psychiatry and Related Sciences, 34, 174-178.

Baron-Cohen, S.,Wheelwright, S., Hill, J., Raste, Y., \& Plumb, I., (2001). The "Reading theMind in the Eyes" test revised version: A study with normal adults, and adults with Asperger syndrome or high-functioning autism. Journal of Child Psychology and Psychiatry, 42, 241-251. 
Baron-Cohen, S., \& Wheelwright, S. (2004) The Empathy Quotient: An Investigation of Adults with Asperger Syndrome or High Functioning Autism, and Normal Sex Differences Journal of Autism and Developmental Disorders, 34, 163-175.

Beck, A., Baruchb, E., Balterb, J.M, Steerb, R., \& Warmana, D. (2004) A new instrument for measuring insight: the Beck Cognitive Insight Scale. Schizophrenia Research, 68, 319-329

Behere, R. V., Raghunandan, V., Venkatasubramanian, G., Subbakrishna, D. K., Jayakumar, P. N., \& Gangadhar, B. N. (2008). Trends- A tool for recognition of emotions in neuropsychiatric disorders. Indian Journal of Psychological Medicine, 30, 32-38. doi: $10.4103 / 0253-7176.43132$

Bell, M. D., Bryson, G. J., \& Lysaker, P. H. (1997). Positive and negative affect recognition in schizophrenia: A comparison with substance abuse and normal control subjects. Psychiatry Research, 73, 73-82.

Bell, M.D., Tsang, H., Greig, T., \& Bryson, G. (2009) Neurocognition, social cognition, perceived social discomfort ando vocational outcomes in schizopheria. Schizophrenia Bulletin, 35, 738-747.

Bell, M. D., Fiszdon, J. M., Greig, T. C., \& Wexler, B.E. (2010). Social attribution test-multiple choice (SAT-MC) in schizophrenia: comparison with community sample and relationship to neurocognitive, social cognitive and symptom measures. Schizophrenia Research, 122, 164-171.

Bell, M. D., Fiszdon, J. M., Greig, T.C., \& Wexler, B.E. (2010). Social attribution test-multiple choice (SAT-MC) in schizophrenia: comparison with community sample and relationship to neurocognitive, social cognitive and symptom measures. Schizophrenia Research, 122, 164-171.

Biagianti, B., Fisher, M., Howard, L., Rowlands, A., Vinogradov, S., \& Woolley, J. (2017) Feasibility and preliminary efficacy of remotely delivering cognitive training to people with schizophrenia using tablets. Schizophrenia Research: Cognition, 10, 7-14. doi:10.1016/j. scog.2017.07.003

Billeke P., \& Aboitiz F. (2013). Social cognition in schizophrenia: from social stimuliprocessingto social engagement. Front Psychiatry, 4, 1-12.

Birchwood, M., Smith, J., Cochrane, R., Wetton, S., \& Copestake, S. (1990) The Social Functioning Scale. The development and validation of a new scale of social adjustment for use in family intervention programmes with schizophrenic patients. The British Journal of Psychiatry, 157, 853-9

Bo, Q., Mao, Z., Li, X., Wang, Z., Wang, C., \& Ma, X. (2017). Use of the MATRICS consensus cognitive battery (MCCB) to evaluate cognitive deficits in bipolar disorder: A systematic review and meta-analysis. PLOS ONE, 12, 1-13.

Bora, E., \& Pantelis, C. (2013). Theory ofmind impairments in first-episode psychosis, individuals at ultra-high risk for psychosis and in first-degree relatives of schizophrenia: systematic review and meta-analysis. Schizophrenia Research, 144, (1-3), 31-36. doi:10.1016/j. schres.2012.12.013.

Brekke, J. S., Hoe, M., Long, J., \& Green, M. F. (2007). How neurocognition and social cognition influence functional change during community-based psychosocial rehabilitation for individuals with schizophrenia. Schizophrenia Bulletin, 33, 1247-1256.

Brown, E. C., Tas, C., Can, H. Esen-Danaci, A., \& Brüne, M. (2014) A closer look at the relationship between the subdomains of social functioning, social cognition and symptomatology in clinically stable patients with schizophrenia. Comprehensive Psychiatry, 55, 25-32.

Browne, J., Penn, D.L., Raykov, T., Pinkham, A.E., Kelsven, S., Buck, B., \& Harvey, P.D., (2016). Social cognition in schizophrenia: factor structure of emotion processing and theory of mind. Psychiatry Research, 242, 150-156.

Brüne, M. (2005) Emotion recognition, 'theory of mind' and social behaviour in schizophrenia. Psychiatry Research, 133, 135-147.

Buck, B., \& Penn, D. L. (2015). Lexical characteristics of emotional narratives in schizophrenia: Relationships with symptoms, functioning, and social cognition. Journal of Nervous and Mental Disease, 203, 702-708. doi:10.1097/NMD.0000000000000354

Buck, K.D., McLeod, H.J., Gumley, A., Dimaggio, G., Buck, B.E., Minor, K.S., \& Lysaker, P.H. (2014). Anhedonia in prolonged schizophrenia spectrum patients with relatively lower vs. higher levels of depression disorders: associations with deficits in social cognition and metacognition. Consciousness and Cognition, 29, 68-75

Carretero-Dios, H., \& Pérez, C. (2007). Standarts for the development and review of instrumental studies: Considerations about test selection in psychological research. International Journal of Clinical and Health Psychology, 7, 863-882

Charernboon, T., \& Patumanond, J. (2017). Social cognition in schizophrenia. Mental Illness, 9, (1), 16-19. doi: 10.4081/mi.2017.7054

Constantino, J. N, Davis, S., Todd, R., Schindler, M., Gross, M., Brophy, S., Metzger, L., Shoushtari, Ch., Splinter, R., \& Reich, W. (2003) Validation of a Brief Quantitative Measure of Autistic Traits: Comparison of the Social Responsiveness Scale with the Autism Diagnostic Interview-Revised. Journal of Autism and Developmental Disorders, 33, 427-433.

Combs, D. R., Adams, S. D., Penn, D. L., Roberts, D., Tiegreen, J., \& Stem, P. (2007). Social cognition and interaction training (SCIT) for inpatients with schizophrenia spectrum disorders: Preliminary findings. Schizophrenia Research, 91, 112-116. doi:10.1016/j. schres.2006.12.010 
Combs, D.R., Penn, D.L., Wicher, M., \& Waldheter, E. (2007). The Ambiguous Intentions Hostility Questionnaire (AIHQ): a new measure for evaluating hostile social-cognitive biases in paranoia. Cognitive Neuropsychiatry, 12, 128-143.

Corcoran, R., \& Frith, C.D. (2005). Thematic reasoning and theory of mind. Accounting for social inference difficulties in schizophrenia. Evolutionary Psychology, 3, 1-19.

Crowne, D. P., \& Marlowe, D. (1960). A new scale of social desirability independent of psychopathology. Journal of Counseling Psychology, 24, 349-354.

Davis, M. C., Lee, J., Horan, W. P., Clarke, A. D., McGee, M. R., Green, M. F., \& Marder, S. R. (2013). Effects of single dose intranasal oxytocin on social cognition in schizophrenia. Schizophrenia Research, 147, 393-397. doi:10.1016/j.schres.2013.04.023

Davis, M. H. (1983). Measuring individual differences in empathy: evidence for a multidi-mensional approach. Journal of Personality and Social Psychology, 44, 113-126.

de Achával, D., Villarreal, M. F., Costanzo, E. Y., Douer, J., Castro, M. N., Mora, M. C., \& Guinjoan, S. M. (2012). Decreased activity in right-hemisphere structures involved in social cognition in siblings discordant for schizophrenia. Schizophrenia Research, 134, 171-179. doi:10.1016/j.schres.2011.11.010

Derntl, B., Seidel, E. M., Schneider, F., \& Habel, U. (2012). How specific are emotional deficits? A comparison of empathic abilities in schizophrenia, bipolar and depressed patients. Schizophrenia Research,142, 58-64.

Dickinson, D., Bellack, A. S., \& Gold, J. M. (2007). Social/communication skills, cognition, and vocational functioning in schizophrenia. Schizophrenia Bulletin, 33, 1213-1220. doi:10.1093/schbul/sb1067.

Donohoe, G., Duignan, A., Hargreaves, A., Morris, D. W., Rose, E., Robertson, D., \& Corvin, A. (2012). Social cognition in bipolar disorder versus schizophrenia: Comparability in mental state decoding deficits. Bipolar Disorders, 14, 743-748. doi:10.1111/bdi.12011

Dziobek, I., Fleck, S., Kalbe, E., Rogers, K., Hassenstab, J., Brand, M., Kessler, J.,Woike, J.K., Wolf, O.T., \& Convit, A. (2006). Introducing MASC: a movie for the assessment of social cognition. Journal of Autism and Developmental Disorders, 36, $623-636$.

Ekman, P., \& Friesen, W. (1976). Pictures of facial affect. Palo Alto, CA:Consulting Psychologists Press.

Elsawy H., El-Hay M.A., \& Badawy, A. (2010) Cognitive-Functions in First Episode Psychosis. Current Psychiatry, 17, 21-7

Eşsizoğlu, A., Köşger, F., Akarsu, F.Ö., Özaydin, Ö., \& Güleç, G. (2017) Theory of mind and selective attention, response inhibition, cognitive flexibility in patients with schizophrenia. Noropsikiyatri Arsivi, 54, 62-167. doi:10.5152/npa.2016.12750

Fanning, J. R., Bell, M. D., \& Fiszdon, J. M. (2012). Is it possible to have impaired neurocognition but good social cognition in schizophrenia? Schizophrenia Research, 135, 68-71. doi:10.1016/j.schres.2011.12.009

Fernandez, A. M., Dufey, M., \& Kramp, U. (2011). Testing the psychometric properties of the interpersonal reactivity index (IRI) in Chile: Empathy in a different cultural context. European Journal of Assessment, 27, 179-185. doi: 10.1027/ 1015-5759/a000065

Fernández-Gonzalo, S., Turon, M., Jodar, M., Pousa, E., Hernandez Rambla, C., García, R., \& Palao, D. (2015). A new computerized cognitive and social cognition training specifically designed for patients with schizophrenia/schizoaffective disorder in early stages of illness: A pilot study. Psychiatry Research, 228, 501-509. doi:10.1016/j.psychres.2015.06.007

Fiszdon, J. M., Richardson, R., Greig, T., \& Bell, M. D. (2007). A comparison of basic and social cognition between schizophrenia and schizoaffective disorder. Schizophrenia Research, 91,117-121. doi:10.1016/j.schres.2006.12.012

Frajo- Apor, B., Pardeller, S., Kemmler, G., Welte, A., \& Hofer, A. (2016). Emotional intelligence deficits in schizophrenia: The impact of non-social cognition. Schizophrenia Research, 172, 131-136. doi:10.1016/j.schres.2016.02.027

Fuentes, I., García, S., Ruíz, J. C., Dasí, C., \& Soler, M. J. (2011). The social cognition scale (SCS): Pilot study about the assessment of social cognition in people with schizophrenia. [La Escala de Cognicioń Social (ECS): Estudio piloto sobre la evaluación de la cognicíon social en las personas con esquizofrenia]. Rehabilitacion Psicosocial, 8, 2-7.

Gagen, E., Gibson, C., Elliot, T., Smedley,K., Jarskog, L., Pedersen, C., \& Penn, D.(2017) Are social skill and empathy separable constructs? A psychometric evaluation of a new role play assessment of empathy for individuals with schizophrenia. Journal of Experimental Psychopathology, 8(2), 175-191.

García, H., Tirapu, J., \& López-Goñi, J. J. (2012). Valoración de la cognición social en esquizofrenia a través del test de la mirada. Implicaciones para la rehabilitación. Avances en Psicología Latinoamericana, 30, 39-51.

Gard, D. E., Fisher, M., Garrett, C., Genevsky, A., \& Vinogradov, S. (2009). Motivation and its relationship to neurocognition, social cognition, and functional outcome in schizophrenia. Schizophrenia Research, 115, 74-81. doi:10.1016/j.schres.2009.08.015.

Gibson, C. M., Penn, D. L., Smedley, K. L., Leserman, J., Elliott, T., \& Pedersen, C. A. (2014). A pilot six-week randomized controlled trial of oxytocin on social cognition and social skills in schizophrenia. Schizophrenia Research, 156, 261-265. doi:10.1016/j.schres.2014.04.009

Gil-Sanz, D., Fernández-Modamio, M., Bengochea-Seco, R., Arrieta-Rodríguez, M., González-Fraile, E., Pérez-Fuentes,...\& Santos-Zorrozúa, B. (2017). ERAT: A new tool to assess basic emotions recognition and its application in schizophrenia. Revista de Psicopatologia y Psicologia Clinica, 22 (2), 85-93.

Gonzalez de Dios, J., Buñuel Alvarez, J.C., Aparicio Rodrigo, M. (2011) Listas guía de comprobación de revisions sistemáticas y meta- 
análisis: Declaración PRISMA. EvidPediatr, 7, 1-6.

Goodman S., Sewell D., Cooley E., \& Leavitt N (1993): Assessing levels of adaptive functioning: The Role Functioning Scale. Community Ment Health Journal, 29,119-131.

Guastella, A. J., Ward, P. B., Hickie, I. B., Shahrestani, S., Hodge, M. A. R., Scott, E. M., \& Langdon, R. (2015). A single dose of oxytocin nasal spray improves higher-order social cognition in schizophrenia. Schizophrenia Research, 168, 628-633. doi:10.1016/j. schres.2015.06.005

Hallerback, M.U., Lugnegard, T., Hjarthag, F., \& Gillberg, C.(2009). The Reading the Mind in the Eyes Test: test-retest reliability of a Swedish version. Cognitive neuropsychiatry, 14, 127-143.

Hasson- Ohayon, I., Avidan-Msika, M., Mashiach-Eizenberg, M., Kravetz, S., Rozencwaig, S., Shalev, H., \& Lysaker, P. H. (2015). Metacognitive and social cognition approaches to understanding the impact of schizophrenia on social quality of life. Schizophrenia Research, 161, 386-391. doi:10.1016/j.schres.2014.11.008

Healey, K., Roberts, D., Combs, D., \& Penn, D. (2015), Observable Social Cognition: A Rating Scale: An interview-based assessment for schizophrenia. Cognitive neuropsychiatry, 20(3), 198-221.

Hogarty, G., Flesher, S., Ulrich, R., Carter, M., Greenwald, D., Pogue-Geile M, Zoretich, R. (2004) Cognitive enhancement therapy for schizophrenia: effects of a 2-year randomized trial on cognition and behavior. Archives of General Psychiatry, 61, 866-76.

Horan, W. P., Green, M. F., Degroot, M., Fiske, A., Hellemann, G., Kee, K., \& Nuechterlein, K. H. (2012). Social cognition in schizophrenia, part 2: 12-month stability and prediction of functional outcome in first-episode patients. Schizophrenia Bulletin, 38, 865872. doi:10.1093/schbul/sbr001

Horton, H. K., \& Silverstein, S. M. (2008). Social cognition as a mediator of cognition and outcome among deaf and hearing people with schizophrenia. Schizophrenia Research, 105, 125-137. doi:10.1016/j.schres.2008.07.003

Inchausti, F., García-Poveda, N.V., Prado-Abril, J., Ortuño-Sierra, J., \& Gaínza-Tejedor, I. (2017). Entrenamiento en habilidades sociales orientado a la metacognición (MOSST): Marco teórico, metodología de trabajo y descripción del tratamiento para pacientes con esquizofrenia. Papeles del Psicólogo, 38, 204-215

Kalin, M., Kaplan, S., Gould, F., Pinkham, A. E., Penn, D. L., \& Harvey, P. D. (2015). Social cognition, social competence, negative symptoms and social outcomes: Inter-relationships in people with schizophrenia. Journal of Psychiatric Research, 68, 254-260. doi:10.1016/j.jpsychires.2015.07.008

Kerr, S. L., \& Neale, J. M. (1993). Emotion perception in schizophrenia: specific deficit or further evidence of generalized poor performance? Journal of Abnormal Psychology, 102, 312-318.

Keith, R. A., Granger, C. V., Hamilton, B. B., \& Fielder, R. C. (1987) The functional independence measure: a new tool for rehabilitation. En Eisenberg. M.. \& Grzesiak, R. (Eds.), In Advances in Clinical Rehabilitation, pp. 6-18. New York: Springer-Verlag.

Kinderman, P., \& Bentall, R.P. (1996). A new measure of causal locus: the internal, personal and situational attributions questionnaire. Personality and Individual Differences, 20, 261-264.

Köther, U., Vettorazzi, E., Veckenstedt, R., Hottenrott, B., Bohn, F., Scheu, F., Pfueller, U., Roesch-Ely, D., \& Moritz, S. (2017). Bayesian Analyses of the Effect of Metacognitive Training on Social Cognition Deficits and Overconfidence in Errors. Journal of Experimental Psychopathology, 8, 158-174.

Kocsis-Bogár, K., Kotulla S., Maier, S., Voracek, M., \& Hennig-Fast, K. (2017). Cognitive correlates of different mentalizing abilities in individuals with high and low trait schizotypy: findings from an extreme-group design. Frontiers in psychology, 8, 922.

Lavoie, M., Plana, I., Jackson, P. L., Godmaire-Duhaime, F., Bédard Lacroix, J., \& Achim, A. M. (2014). Performance in multiple domains of social cognition in parents of patients with schizophrenia. Psychiatry Research, 220, 118-124. doi:10.1016/j.psychres.2014.07.055

Lin, C.H., Huang, C.L., Chang, Y.C., Chen, P.W., Lin, C.Y., Tsai, G.E., \& Lane, H.Y. (2013). Clinical symptoms, mainly negative symptoms, mediate the influence of neurocognition and social cognition on functional outcome of schizophrenia. Schizophrenia Research, 146(1-3), 231-237. doi: 10.1016/j.schres.2013.02.009.

Lindenmayer, J., McGurk, S. R., Khan, A., Kaushik, S., Thanju, A., Hoffman, L., \& Herrmann, E. (2013). Improving social cognition in schizophrenia: A pilot intervention combining computerized social cognition training with cognitive remediation. Schizophrenia Bulletin, 39, 507-517. Recuperado desde www.scopus.com

Linke, M., Jankowski, K. S., Ciołkiewicz, A., Jedrasik-Styła, M., Parnowska, D., Gruszka, A., \& Wichniak, A. (2015). Age or age at onset? which of them really matters for neuro and social cognition in schizophrenia? Psychiatry Research, 225, 197-201. doi:10.1016/j. psychres.2014.11.024

Lugnegård, T., Unenge Hallerbäck, M., Hjärthag, F., \& Gillberg, C. (2013). Social cognition impairments in asperger syndrome and schizophrenia. Schizophrenia Research, 143, 277-284. doi:10.1016/j.schres.2012.12.001

Lysaker, P. H., Ringer, J. M., Buck, K. D., Grant, M., Olesek, K., Leudtke, B. L., \& Dimaggio, G. (2012). Metacognitive and social cognition deficits in patients with significant psychiatric and medical adversity: A comparison between participants with schizophrenia 
and a sample of participants who are HIV-positive. Journal of Nervous and Mental Disorders, 200, 130-134.

Lysaker, P., Dimaggio, G., Carcione, A., Procacci, M., Buck, K., Davis, L., Nicolò, G., (2010). Metacognition and schizophrenia: the capacity for self-reflectivity as a predictor for prospective assessments of work performance over six months. Schizophrenia Research, 122, 124-130.

Lysaker, P., Gumley, A., Luedtke, B., Buck, K., Ringer, J., Olesek, K., Dimaggio, G., (2013). Social cognition and metacognition in schizophrenia: evidence of their independence and linkage with outcomes. Acta Psychiatrica Scandinavica, 127, $239-247$.

Lysaker, P., Vohs, J., Ohayon, I., Kukla, M., Wierwille, J., \& Dimaggio, G., (2013). Depression and insight in schizophrenia: comparisons of levels of deficits in social cognition and metacognition and internalized stigma across three profiles. Schizophrenia Research. $148,18-23$.

Lysaker, P., Vohs, J., \& Tsai, J. (2009). Negative symptoms and concordant impairments in attention in schizophrenia: associations with social functioning, hope, selfesteem and internalized stigma. Schizophrenia Research, 110, 165-172

Maat, A., Fett, A., \& Derks, E. (2012) Social cognition and quality of life in schizophrenia. Schizophrenia Research, 137, 212-8. http:// dx.doi.org/10.1016/j.schres.2012.02.017.

Maat, A., Cahn, W., Gijsman, H. J., Hovens, J. E., Kahn, R. S., \& Aleman, A. (2014). Open, randomized trial of the effects of aripiprazole versus risperidone on social cognition in schizophrenia. European Neuropsychopharmacology, 24, 575-584. doi:10.1016/j. euroneuro.2013.12.009.

Martín, M., Secades, R., López-Goñi, J., \& Tirapu., J. (2017). Empatía, cognición social y calidad de vida subjetiva en esquizofrenia. Anales del Sistema Sanitario de Navarra, 40, 211-219.

Martínez- Domínguez, S., Penadés, R., Segura, B., González-Rodríguez, A., \& Catalán, R. (2015). Influence of social cognition on daily functioning in schizophrenia: Study of incremental validity and mediational effects. Psychiatry Research, 225, 374-380. doi:10.1016/j. psychres.2014.12.011

Matsumoto, Y., Takahashi, H., Murai, T., \& Takahashi, H. (2015). Visual processing and social cognition in schizophrenia: Relationships among eye movements, biological motion perception, and empathy. Neuroscience Research, 90, 95-100. doi:10.1016/j.neures.2014.10.011.

Mayer, J. D., Salovey, P., \& Caruso, D. R., (2002). Mayer-Salovey-Caruso Emotional Intelligence Test (MSCEIT) User's Manual. Toronto, Canada: Multi-Health Systems.

Murray, H. (1943). Manual for the Thematic Apperception Test. Cambridge, MA: Harvard University Press.

McDonald, S. (2017). What's New in the Clinical Management of Disorders of Social Cognition?. Brain Impairment, 18, 2-10.

McDonald, S., Flanagan, S., Rollins, J., \& Kinch, J., (2003). TASIT: a new clinical tool for assessing social perception after traumatic brain injury. Journal of Head Trauma Rehabilitation, 18, 219-238.

Mehta U. M., Thirthalli J., Basavaraju R., \& Gangadhar B. N. (2014). Association of intracortical inhibition with social cognition deficits in schizophrenia: findings from a transcranial magnetic stimulation study. Schizophrenia Research, 158, 146-150. 10.1016/j. schres.2014.06.043

Mehrabian, A. (1996) Manual for BalancedEmotionalEmpathyScale (BEES). Monterey CA: Albert Meherabian

Mervis, J.E., Capizzi, R.J., Boroda, E., \& Macdonald, A.W. (2017) Transcranial direct current stimulation over the dorsolateral prefrontal cortex in schizophrenia: A quantitative review of cognitive outcomes. Frontiers in Human Neuroscience, 11(44).

Minor, K. S., Marggraf, M. P., Davis, B. J., Luther, L., Vohs, J. L., Buck, K. D., \& Lysaker, P. H. (2015). Conceptual disorganization weakens links in cognitive pathways: Disentangling neurocognition, social cognition, and metacognition in schizophrenia. Schizophrenia Research, 169, 153-158. doi:10.1016/j.schres.2015.09.026

Minor, K.S., \& Lysaker, P.H. (2014). Necessary, but not sufficient: links between neurocognition, social cognition, and metacognition in schizophrenia are moderated by disorganized symptoms. Schizophrenia Research , 159, 198-204.

Miyata, J., Yamada, M., Namiki, C., Hirao, K., Saze, T., Fujiwara, H., \& Murai, T. (2010). Reduced white matter integrity as a neural correlate of social cognition deficits in schizophrenia. Schizophrenia Research, 119, 232-239. doi:10.1016/j.schres.2009.12.

Moritz, S., Andreou, C., Schneider, B., Wittekinda, C., Menon, M., Balzand, R., \& Woodward, T., (2014) Sowing the seeds of doubt: a narrative review on metacognitive trainingin schizophrenia. Clinical Psychology Review, 34, 358-366.

Mortiz, S., \& Woodwward, TS. (2007). Metacognitive training in schizophrenia: from basic research to knowledge translation and intervention. Current Opinion in Psychiatry, 20(6), 619-625. doi: 10.1097/YCO.0b013e3282f0b8ed

Murray, H. (1943). Manual for the Thematic Apperception Test. Cam- bridge, MA: Harvard University Press.

Nitzburg, G. C., Burdick, K. E., Malhotra, A. K., \& DeRosse, P. (2015). Social cognition in patients with schizophrenia spectrum and bipolar disorders with and without psychotic features. Schizophrenia Research: Cognition, 2, 2-7. doi:10.1016/j.scog.2014.12.003

Nuechterlein, K.H., Green, M.F., Kern, R.S., Baade, L.E., Barch, D.M., Cohen, J. D., ...Marder, S.R. (2008). The MATRICS Consensus Cognitive Battery, part 1: test selection, reliability, and validity. The American Journal of Psychiatry, 165, 203-13. doi: 10.1176/appi. ajp.2007.07010042. 
Okruszek, Ł., Bala, A., Wordecha, M., Jarkiewicz, M., Wysokiński, A., Szczepocka, E., Piejka, A., Zaborowska, O., Szantroch, M., Rysz, A., \& Marchel, A. (2017). Social cognition in neuropsychiatric populations: a comparison of theory of mind in schizophrenia and mesial temporal lobe epilepsy. Scientific reports, 7, 484.

Olszewski, A., Kikinis, Z., Gonzalez, C., Coman, I., Makris, N., Gong, X., Rathi, Y., Zhu, A., Antshel, K., Fremont, W., Kubicki, M., Bouix, S., Shenton, M., \& Kates, W. (2017). The social brain network in 22q11.2 deletion syndrome: a diffusion tensor imaging study. Behavioral and brain functions, 13(4), 1-17.

Palumbo, D., Mucci, A., Piegari, G., D’Alise, V., Mazza, A., \& Galderisi, S. (2017)SoCIAL - training cognition in schizophrenia: A pilot study. Neuropsychiatric Disease and Treatment, 13, 1947-1956. doi: 10.2147/NDT.S136732

Patterson, T. L., Moscona, S., McKibbin, C. L., Davidson, K., \& Jeste, D. V. (2001). Social skills performance assessment among older patients with schizophrenia. Schizophrenia Research, 48, 351-360.

Peterson, C., Semmel, A., von Bayer, C., Abramson, L. Y., Metalsky, G. I. \&, Seligman, M.E.P. (1982). The Attributional Style Questionnaire. Cognitive Therapyand Research, 6, 287-300.

Pinkham, A., Penn, D., Green, M., Buck, B., Healey, K.., \& Harvey, P. (2014). The social cognition psychometric evaluation study: results of the expert survey and RAND panel. Schizophrenia Bulletin, 40, 813-823. doi: 10.1093/schbul/sbt081

Schizophr. Bull., 40 (2014), pp. 813-823Poole, J. H., Tobias, F. C., \& Vinogradov, S. (2000). The functional relevance of affect recognition errors in schizophrenia. Journal of the International Neuropsychological Society, 6, 649-658.

Rassovsky, Y., Dunn, W., Wynn, J., Wu, A. D., Iacoboni, M., Hellemann, G., \& Green, M. F. (2015). The effect of transcranial direct current stimulation on social cognition in schizophrenia: A preliminary study. Schizophrenia Research, 165, 171-174. doi:10.1016/j. schres.2015.04.016.

Renard, S. B., Pijnenborg, M., \& Lysaker, P. H. (2012). Dissociation and social cognition in schizophrenia spectrum disorder. Schizophrenia Research, 137, 219-223. doi:10.1016/j.schres.2012.02.001.

Reeder, C., Huddy, V., Cella, M., Taylor, R., Greenwood, K., Landau, S., \& Wykes, T. (2017). A new generation computerised metacognitive cognitive remediation programme for schizophrenia (CIRCuiTS): a randomised controlled trial. Psychological medicine, 47, 2720-2730.

Roberts, D. L., \& Penn, D. L. (2009). Social cognition and interaction training (SCIT) for outpatients with schizophrenia: A preliminary study. Psychiatry Research, 166, 141-147. doi:10.1016/j.psychres.2008.02.007

Roberts, D. L., Combs, D. R., Willoughby, M., Mintz, J., Gibson, C., Rupp, B., \& Penn, D. L. (2014). A randomized, controlled trial of social cognition and interaction training (SCIT) for outpatients with schizophrenia spectrum disorders. British Journal of Clinical Psychology, 53, 281-298. doi:10.1111/bjc.12044.

Robertson D. A., Hargreaves, A., Kelleher, E. B., Morris, D., Gill, M., Corvin, A., \& Donohoe, G. (2013). Social dysfunction in schizophrenia: An investigation of the GAF scale's sensitivity to deficits in social cognition. Schizophrenia Research, 146, 363-365. doi:10.1016/j.schres.2013.01.016

Rocha, N. B. F., \& Queirós, C. (2013). Metacognitive and social cognition training (MSCT) in schizophrenia: A preliminary efficacy study. Schizophrenia Research, 150, 64-68. doi:10.1016/j.schres.2013.07.057.

Rodríguez Sosa, J. T., Gil Santiago, H., Trujillo Cubas, A., Winter Navarro, M., León Pérez, P., Guerra Cazorla, L. M., \& Martín Jiménez, J. M. (2013). Social cognition in patients with schizophrenia, their unaffected first degree relatives and healthy controls. comparison between groups and analysis of associated clinical and sociodemographic variables. Revista De Psiquiatria y Salud Mental, 6, 160167. doi:10.1016/j.rpsm.2012.11.003

Russell, T. A., Green, M. J., Simpson, I., \& Coltheart, M. (2008). Remediation of facial emotion perception in schizophrenia: concomitant changes in visual attention. Schizophrenia Research, 103, 248-256. http://dx.doi.org/10.1016/j.schres.2008.04.033.

Santelices, M., Zapata, J., Fischersworring, M., Pérez, F., Mata, C., Barco, B., Olhaberry,M., \& Farkas, Ch. (2016). Intervenciones Basadas en la Mentalización para Padres y Educadores: Una Revisión Sistemática. Terapia Psicológica, 34, 71-80.

Savla, G.N., Vella, L., Armstrong, C.C., Penn, D.L., \& Twamley, E.W.(2012). Deficits in domains of social cognition in schizophrenia: a meta-analysis of the empirical evidence. Schizophrenia Bulletin, 39, 979-992.

Schwarzer, R. (2008), Modeling Health Behavior Change: How to Predict and Modify the Adoption and Maintenance of Health Behaviors. Applied Psychology, 57: 1-29. doi:10.1111/j.1464-0597.2007.00325.x

Schimansky J., David, N., Rössler, W., \& Haker, H. (2010). Sense of agency and mentalizing: Dissociation of subdomains of social cognition in patients with schizophrenia. Psychiatry Research, 178, 39-45. doi:10.1016/j.psychres.2010.04.002

Sergi, M.J., Fiske, A.P., Horan, W.P., Kern, R.S., Kee, K.S., Subotnik, K.L.,... Green, MF. (2009). Development of a measure of relationship perception in schizophrenia. Psychiatry Research, 166(1), 54-62.

Sparks, A., McDonald, S., Lino, B., O’Donnell, M., \& Green, M. J. (2010). Social cognition, empathy and functional outcome in schizophrenia. Schizophrenia Research, 122, 172-78. http://dx.doi.org/10.1016/j.schres.2010.06.011 Research support, non-U.S. gov't. 
Stone, V. E., Baron-Cohen, S., \& Knight, R. T. (1998). Frontal lobe contributions to theory of mind. Journal of Cognitive Neuroscience, 10, 640-656.

Sung-Wan, K., Bong Ju, L., Jung Jin, K., Je-Chun, Y., Kyu Young, L., Seung-Hee, W., Seung-Hwan, L., Seung-Hyun, K., Shi Hyun, K., \& Young-Chul, C. (2017). Design and Methodology of the Korean Early Psychosis Cohort Study. Psychiatry Investigation, 14, 93-99

Tas C., Danaci, A. E., Cubukcuoglu, Z., \& Brüne, M. (2012). Impact of family involvement on social cognition training in clinically stable outpatients with schizophrenia - A randomized pilot study. Psychiatry Research, 195, 32-38. doi:10.1016/j.psychres.2011.07.031

Thaler N. S., Sutton, G. P., \& Allen, D. N. (2014). Social cognition and functional capacity in bipolar disorder and schizophrenia. Psychiatry Research, 220, 309-314. doi:10.1016/j.psychres.2014.08.035.

Torgalsbøen, A., Mohn, C., \& RishovdRund, B. (2014). Neurocognitive predictors of remission of symptoms and social and role functioning in the early course of first-episode schizophrenia. Psychiatry Research, 216, 1-5. Recuperado desde www.scopus.com

Tso I. F., Grove, T. B., \& Taylor, S. F. (2010). Emotional experience predicts social adjustment independent of neurocognition and social cognition in schizophrenia. Schizophrenia Research, 122, 156-163. doi:10.1016/j.schres.2009.12.007

Urrútia, G., \& Bonfill, X. (2010) Declaración PRISMA: una propuesta para mejorar la publicación de revisiones sistemáticas y metaanálisis. Medicina Clínica Barcelona, 135, 507-511

Vázquez- Campo, M., Maroño, Y., Lahera, G., Mateos, R., \& García-Caballero, A. (2016). E-motional training®: Pilot study on a novel online training program on social cognition for patients with schizophrenia. Schizophrenia Research: Cognition, 4, 10-17. doi:10.1016/j.scog.2015.11.007

Vendittelli N, Veltro F, Oricchio I, Cappuccini, M., Roncone, R., \& Simonato, P. (2003). L'intervento cognitivo comportamental en el Servizio Psichiatrico di Diagnosi e Cura. Torino: Centro Scientifico Editore.

Wang, Y., Roberts, D. L., Xu, B., Cao, R., Yan, M., \& Jiang, Q. (2013). Social cognition and interaction training for patients with stable schizophrenia in chinese community settings. Psychiatry Research, 210, 751-755. doi:10.1016/j.psychres.2013.08.038

Westen, D., Klepser, J., Ruffins, S., Silverman, M., Lifton, N., \& Boekamp, J. (1991). Object relations in childhood and adolescence: The development of working representations. Journal of Consulting and Clinical Psychology, 59, 400-409.

Wiig, E. H., Semel, E., \& Secord, W. A. (2013). Clinical evaluations of language fundamentals-fifth edition (CELF-5). Bloomington, MN: NCS Pearson.

White, R., Laithwaite, H., \& Gilbert, P. (2013). Negative symptoms in schizophrenia: The role of social defeat. Recuperado desde www. scopus.com

Whitton, A. E., \& Henry, J. D. (2013). The relationship between sub-clinical obsessive-compulsive symptoms and social cognition in chronic schizophrenia. British Journal of Clinical Psychology, 52, 115-128. doi:10.1111/bjc.12004

Woolley, J. D., Chuang, B., Lam, O., Lai, W., O’Donovan, A., Rankin, K. P., \& Vinogradov, S. (2014). Oxytocin administration enhances controlled social cognition in patients with schizophrenia. Psychoneuroendocrinology, 47, 116-125. doi:10.1016/j.psyneuen.2014.04.024

Wynn, J. K., Sugar, C., Horan, W. P., Kern, R., \& Green, M. F. (2010). Mismatch negativity, social cognition, and functioning in schizophrenia patients. Biological Psychiatry, 67, 940-947. doi:10.1016/j.biopsych.2009.11.024

Yamada M., Hirao, K., Namiki, C., Hanakawa, T., Fukuyama, H., Hayashi, T., \& Murai, T. (2007). Social cognition and frontal lobe pathology in schizophrenia: A voxel-based morphometric study. NeuroImage, 35, 292-298. doi:10.1016/j.neuroimage.2006.10.046

Zabala, M. L., Richard's, M. M., Breccia, F., \& López, M. (2018). Relaciones entre empatía y teoría de la mente en niños y adolescentes. Pensamiento Psicológico, 16, 47-57. Recuperado desde http://www.scielo.org.co/pdf/pepsi/v16n2/1657-8961-pepsi-16-02-47.pdf

Zhang, B., Han, M., Tan, S., Yang, F., Tan., Y., Jiang, S., Zhang, X., \& Huang, X. (2017). Gender differences measured by the MATRICS consensus cognitive battery in chronic schizophrenia patients. Scientific Reports, 7(1), 1-8. 